$\mathrm{MZ}-\mathrm{TH} / \mathbf{0 2 - 1 0}$

\title{
One-loop corrections to four-point functions with two external massive fermions and two external massless partons.
}

\author{
J.G. Körner ${ }^{a \dagger}$ and Z. Merebashvili $b, \ddagger$ \\ a. Institut für Physik, Johannes Gutenberg-Universität, D-55099 Mainz, Germany \\ b. High Energy Physics Institute, Tbilisi State University, 380086 Tbilisi, Georgia
}

\begin{abstract}
We present a complete set of one-loop matrix elements relevant for the hadroproduction of heavy quarks in next-to-leading order employing dimensional regularization to isolate ultraviolet and soft divergences. All results of the perturbative calculation are given in detail. These one-loop matrix elements can also be used as input in the determination of the corresponding next-to-leading order cross sections for heavy flavor photoproduction and in photon-photon reactions, as well as for any of the relevant crossed processes. Our results are tested against the results of other related studies in which unpolarized and longitudinally polarized processes were considered.
\end{abstract}

PACS number(s): 12.38.Bx, 13.85.-t, 13.85.Fb, 13.88.+e

\footnotetext{
†e-mail address: koerner@thep.physik.uni-mainz.de

$\ddagger$ e-mail address: mereb@sun20.hepi.edu.ge
} 


\section{INTRODUCTION}

The production of heavy quarks is important for our understanding of nature. Intensive experimental studies of a heavy quark production in various reactions involving unpolarized initial particles are presently being carried out. However, not less important are analogous studies when initial particles are polarized, either longitudinally or transversely. Experiments on longitudinally polarized initial particles are taking place [1], are being planned [2] and proposed [3]. These will deal with many aspects of polarized reactions, e.g. the problem of the polarized gluon structure function $g_{2}$, the validity of the Drell-Hearn-Gerasimov (DHG) sum rule in QCD, etc...

At the leading order (LO) Born term level, various heavy quark production mechanisms have been studied some time ago. However, the importance of knowing the next-to-leading order (NLO) corrections cannot be overemphasized. Unpolarized NLO corrections for heavy quark hadroproduction were first presented in [4, 5], and in [6, 7] for photoproduction. Corresponding polarized results were calculated in [8] and [9, 10, 11, 12]. In all these papers cross sections were obtained by folding in the Born term matrix elements from the very beginning. Analytical results for the so called "virtual plus soft" terms were presented in [5.,7,10] for the photoproduction and unpolarized hadroproduction of heavy quarks. Complete analytic results for the polarized and unpolarized photoproduction, including real bremsstrahlung, can be found in [12].

Let us emphasize the importance of knowing one-loop matrix elements, which contain the full spin information of the relevant subprocess. When the one-loop contributions are folded with the Born term contributions and spin summed, as in a NLO rate calculation, the information on the spin content of the one-loop contribution is lost and cannot be reconstructed from the rate expressions. On the other hand, having expressions for matrix elements allows one to easily derive the one-loop contributions to partonic cross section including any polarization of the incoming or outgoing particles. Also, it allows one to obtain any of the crossed processes, including the ones with a heavy incoming particle, that are needed for the different versions of variable flavor number schemes, for the direct checking of the DHG sum rule on the partonic level and possibly for doing future parametrizations for structure functions. And all this without doing calculations from the beginning. This work presents detailed results on a NLO calculation of partonic matrix elements for the set of one-loop Feynman graphs present in hadroproduction of heavy flavors, separately for every Feynman diagram in order to facilitate the use of the results for other relevant processes that differ by color factors.

The one-loop results presented in this paper are an important input for part of the nextto-next-to-leading (NNLO) order calculation of the perturbative corrections to heavy flavor production. Last but not least, the imaginary parts of the one-loop amplitudes presented here are a necessary ingredient for the calculation of $T$-odd observables which are known to be fed by the NLO absorptive (or imaginary) parts of the one-loop contributions to a given process. 
The subprocesses that receive one-loop corrections which are considered in this paper proceed through the following two partonic channels:

$$
g+g \rightarrow Q+\bar{Q}
$$

where $g$ denotes a gluon and $Q(\bar{Q})$ denotes a heavy quark (antiquark), and

$$
q+\bar{q} \rightarrow Q+\bar{Q}
$$

where $q(\bar{q})$ is a light massless quark (antiquark).

We note that the Abelian part of the NLO result for (1.1) provides the NLO corrections to heavy flavor production by two on-shell photon collisions

$$
\gamma+\gamma \rightarrow Q+\bar{Q}
$$

with the appropriate color factor substitutions. The results for (1.1) can be also used to determine corresponding amplitudes for heavy flavor photoproduction

$$
\gamma+g \rightarrow Q+\bar{Q}
$$

We mention that the partonic processes (1.1) and (1.2) are needed for the calculation of the contributions of single- and double-resolved photons in the photonic processes (1.3) and (1.4).

Cross sections for the process (1.3) have already been determined in 13, 14, 15 for unpolarized and in [15,16] for polarized initial photons. Note that the authors of [16] used a nondimensional regularization scheme to regularize the poles of divergent integrals. In the papers 13,16] analytic results were presented for "virtual plus soft" contributions alone. We also note that complete analytic results including hard gluon contributions can be found only in [15]. The reaction (1.3) will be investigated at future linear colliders. NLO corrections for the heavy quark production cross section (1.3) are of interest in themselves as they represent an irreducible background to the intermediate Higgs boson searches for Higgs masses in the range of 90 to $160 \mathrm{GeV}$.

The paper is organized as follows. Section II contains an outline of our general approach as well as matrix elements of the gluon fusion subprocess for the self-energy and vertex contributions including their renormalization. In Section III we discuss the oneloop contributions to the four box diagrams in the same gluon-gluon subprocess and give a detailed description of our global checks on gauge invariance for our results. Section IV presents analytic results on the quark-antiquark subprocess (1.2). Whereas Sections II-IV deal with the real (or dispersive) parts of the one-loop amplitudes we turn to discuss their imaginary (or absorptive) parts in Section V. Section V also includes a discussion on how one may obtain the corresponding absorptive parts in different crossed channels. Our main results are summarized in Section VI. Finally, in two Appendices we present results for various coefficient functions completing our determination of NLO matrix elements. 


\section{CONTRIBUTIONS OF THE TWO- AND THREE-POINT FUNCTIONS TO GLUON FUSION}

The Born and the one-loop contributions to the gluon fusion partonic reaction $g\left(p_{1}\right)+$ $g\left(p_{2}\right) \rightarrow Q\left(p_{3}\right)+\bar{Q}\left(p_{4}\right)$ are shown in Figs. 1-3. In this section we discuss our calculation of the self-energy and vertex graphs that contribute to the above subprocess. With the 4-momenta $p_{i}(i=1, \ldots, 4)$ as indicated in the Fig. 1 and with $m$ the heavy quark mass we define:

$$
s \equiv\left(p_{1}+p_{2}\right)^{2}, \quad t \equiv T-m^{2} \equiv\left(p_{1}-p_{3}\right)^{2}-m^{2}, \quad u \equiv U-m^{2} \equiv\left(p_{2}-p_{3}\right)^{2}-m^{2} .
$$

To isolate ultraviolet (UV) and infrared/collinear (IR/M) divergences we have carried out all our calculations in both conventional regularization schemes, namely the standard dimensional regularization scheme (DREG) [17] and the dimensional reduction scheme (DRED) [18]. In what follows, we present results for the DREG, as well as the difference $\Delta=$ DRED-DREG. A brief characterization of the two regularization schemes is the following: In DREG both tensorial structures (e.g. gamma matrices, metric tensors, etc...) and momenta are continued to $n \neq 4$, while in DRED only momenta are continued to $n \neq 4$ whereas the tensorial structures are those of $n=4$.

First of all we note that in general the matrix elements for all the Feynman diagrams in the gluon fusion subprocess are written in the form

$$
M=\epsilon_{\mu}\left(p_{1}\right) \epsilon_{\nu}\left(p_{2}\right) \bar{u}\left(p_{3}\right) M^{\mu \nu} v\left(p_{4}\right),
$$

However, for the purposes of brevity, we will present our results in terms of the amplitudes $M^{\mu \nu}$ omitting the polarization vectors and Dirac spinors. Of course, their presence is implicitly understood throughout this paper in that the mass shell conditions $p_{1}^{\mu} \epsilon_{\mu}\left(p_{1}\right)=0$ and $\not p_{3} u\left(p_{3}\right)=m u\left(p_{3}\right)$ etc. are being used to simplify $M^{\mu \nu}{ }^{1}$. Furthermore, $M^{\mu \nu}$ for all the one-loop graphs considered in this paper contains a common factor due to the one-loop integration. Define the quantity

$$
C_{\varepsilon}\left(m^{2}\right) \equiv \frac{\Gamma(1+\varepsilon)}{(4 \pi)^{2}}\left(\frac{4 \pi \mu^{2}}{m^{2}}\right)^{\varepsilon} .
$$

We will omit from all of our one-loop $M^{\mu \nu}$ amplitudes the common factor

$$
\mathcal{C}=g^{4} C_{\varepsilon}\left(m^{2}\right)
$$

where $g$ is the renormalized coupling constant.

\footnotetext{
${ }^{1}$ According to the discussion in 19 this implies that, when further processing our LO and one-loop results in cross section calculations by folding in the appropriate amplitudes, one may use the Feynman gauge for the spin sums of polarization vectors. At the same time ghost contributions associated with external gluons have to be omitted.
} 
For our analysis of matrix elements it is important to describe various crossed heavy flavor production channels. We make it clear from the outset that an additional u-channel set of graphs, that topologically differ from the t-channel ones, are obtained by interchange of bosonic lines (not momenta). In particular, for calculational purposes, we will always be relating t- and u-channel Feynman diagrams by the following procedure:

$$
\mathcal{M}_{t} \leftrightarrow \mathcal{M}_{u} \equiv\left\{a \leftrightarrow b, \quad p_{1} \leftrightarrow p_{2}, \quad \mu \leftrightarrow \nu\right\}
$$

with $a, b$ color indices of bosons and where all three interchanges are performed simultaneously. Note that the second interchange in (2.5) implies also the interchange $t \leftrightarrow u$, but not vice versa. One case, involving two vertex diagrams, when the above transformation (2.5) does not correspond to "true" u-channel topologies, is discussed below. In general, when speaking about t-u symmetry of given amplitudes, we will imply invariance of those amplitudes under the transformations (2.5).

We start by writing down matrix elements for the leading order Born terms. For the t-channel gluon fusion subprocess (first graph in Fig. 1) we have:

$$
B_{t}^{\mu \nu}=-i T^{b} T^{a} \gamma^{\mu}\left(p_{3}-\not p_{1}+m\right) \gamma^{\nu} / t
$$

where $T^{b}$ and $T^{a}$ are generators $\left(T^{a}=\lambda^{a} / 2, a=1, \ldots, 8\right.$ and the $\lambda^{a}$ are Gell-Mann matrices) that define the fundamental representation of the Lie algebra of the color $\mathrm{SU}(3)$ group. Analogously, for the u- and s-channels we have, respectively,

$$
\begin{aligned}
& B_{u}^{\mu \nu}=-i T^{a} T^{b} \gamma^{\nu}\left(\not p_{3}-\not p_{2}+m\right) \gamma^{\mu} / u, \\
& B_{s}^{\mu \nu}=i\left(T^{a} T^{b}-T^{b} T^{a}\right) C_{3}^{\mu \nu \sigma} \gamma_{\sigma} / s
\end{aligned}
$$

where the tensor $C_{3}^{\mu \nu \sigma}$ is defined according to the Feynman rules for the three-gluon coupling. We have omitted a common factor $g^{2}$ in the Born amplitudes. Acting with Dirac spinors on the above Born matrix elements from the left and the right and using the effective relations $p_{1}^{\mu}=p_{2}^{\nu}=0$, as remarked on before, we arrive at the following expressions for the leading order matrix elements:

$$
\begin{aligned}
& B_{t}^{\mu \nu}=i T^{b} T^{a}\left(\gamma^{\mu} \not p_{1} \gamma^{\nu}-2 p_{3}^{\mu} \gamma^{\nu}\right) / t \\
& B_{u}^{\mu \nu}=i T^{a} T^{b}\left(2 p_{4}^{\mu} \gamma^{\nu}-\gamma^{\nu} \not p_{1} \gamma^{\mu}\right) / u=i T^{a} T^{b}\left(\gamma^{\nu} \not p_{2} \gamma^{\mu}-2 p_{3}^{\nu} \gamma^{\mu}\right) / u \\
& B_{s}^{\mu \nu}=2 i\left(T^{a} T^{b}-T^{b} T^{a}\right)\left(g^{\mu \nu} \not p_{1}+p_{2}^{\mu} \gamma^{\nu}-p_{1}^{\nu} \gamma^{\mu}\right) / s .
\end{aligned}
$$

Next we proceed with the description of the two-point contributions to the matrix element of the subprocess (1.1). But before we turn to the two-point functions one should mention that our choice of renormalization scheme will be a fixed flavor scheme throughout this paper. This implies that we have a total number of flavors $n_{f}=n_{l f}+1$, where $n_{l f}$ is the number of light (e.g. massless) flavors plus one produced heavy flavor, with only $n_{l f}$ light flavors involved/active in the $\beta$ function for the running a QCD coupling $\alpha_{s}$ and in the splitting functions that determine the evolution of the structure functions. When having 
massless particles in the loops we are using the standard $\overline{\mathrm{MS}}$ scheme, while the contribution of a heavy quark loop in the gluon self-energy with on-shell external legs is subtracted out entirely.

Consider first the two t-channel self-energy graphs (2d2) and (2d3) with external legs on-shell (note that in the graph numeration the first number identifies the figure which the given diagram refers to). These graphs are very important as they determine the renormalization parameters in the quark sector. Throughout this paper we use the so called on-shell prescription for the renormalization of heavy quarks, the essential ingredients of which we describe in the following. When dealing with massive quarks one has to choose a parameter to which one renormalizes the heavy quark mass. It is natural to choose a quark pole mass for such a parameter - the only "stable" mass parameter in QCD. The condition on the renormalized heavy quark self-energy $\Sigma_{r}(\not p)$ is

$$
\left.\Sigma_{r}(\not p)\right|_{\not p=m}=0
$$

which removes the singular internal propagator in these self-energy diagrams. The above condition determines the mass renormalization constant $Z_{m}$. For the wave function renormalization we have used the usual condition (see e.g. Ref. [6])

$$
\left.\frac{\partial}{\partial \not p} \Sigma_{r}(\not p)\right|_{\not p=m}=0
$$

which fully determines the wave function renormalization constant $Z_{2}$. Since the condition (2.8) is not mandatory in general, there is a freedom in determining the constant $Z_{2}$. Therefore, we will list our expressions for these constants. In DREG we arrive at

$$
Z_{m}=1-3 g^{2} C_{F} C_{\varepsilon}\left(m^{2}\right)\left(\frac{1}{\varepsilon^{\prime}}+\frac{4}{3}\right), \quad Z_{2}=1-g^{2} C_{F} C_{\varepsilon}\left(m^{2}\right)\left(\frac{1}{\varepsilon^{\prime}}+4+\frac{2}{\varepsilon}\right) .
$$

And in DRED we obtain

$$
Z_{m}=1-3 g^{2} C_{F} C_{\varepsilon}\left(m^{2}\right)\left(\frac{1}{\varepsilon^{\prime}}+\frac{5}{3}\right), \quad Z_{2}=1-g^{2} C_{F} C_{\varepsilon}\left(m^{2}\right)\left(\frac{1}{\varepsilon^{\prime}}+5+\frac{2}{\varepsilon}\right),
$$

where $C_{F}=4 / 3$ and where we use $1 / \varepsilon^{\prime}$ to indicate which terms are of ultraviolet origin. Now we are in a position to write our results for the $(2 \mathrm{~d} 2)$ and $(2 \mathrm{~d} 3)$ quark self-energy diagrams. In DREG we have:

$$
M_{(2 \mathrm{~d} 2)}^{\mu \nu}=M_{(2 \mathrm{~d} 3)}^{\mu \nu}=-C_{F} B_{t}^{\mu \nu}\left(\frac{1}{\varepsilon^{\prime}}+4 \ln \frac{m^{2}-p_{3}^{2}}{m^{2}}\right),
$$

where we clearly see the soft divergence in the logarithm that diverges on the mass-shell. Apparently, this very same logarithm appears also when calculating the wave function renormalization constant $Z_{2}$. This means that on-shell mass renormalization does not actually completely remove the divergent propagator, but rather transforms the strong 
linear divergence to the "softer" logarithmic divergence, which can be dealt with in QCD. Indeed, one can rigoriously prove that there exists the following effective correspondence relation:

$$
\left.\ln \frac{m^{2}-p^{2}}{m^{2}}\right|_{p^{2}=m^{2}} \rightarrow \frac{1}{2 \varepsilon}+\frac{1}{1-2 \varepsilon}=\frac{1}{2 \varepsilon}+1+\mathcal{O}(\varepsilon) .
$$

With this in mind we obtain final results for the two self-energy graphs after mass renormalization was carried out:

$$
\begin{array}{ll}
\text { DREG : } & M_{(2 \mathrm{~d} 2)}^{\mu \nu}=M_{(2 \mathrm{~d} 3)}^{\mu \nu}=-C_{F} B_{t}^{\mu \nu}\left(\frac{1}{\varepsilon^{\prime}}+4+\frac{2}{\varepsilon}\right), \\
\text { DRED : } & M_{(2 \mathrm{~d} 2)}^{\mu \nu}=M_{(2 \mathrm{~d} 3)}^{\mu \nu}=-C_{F} B_{t}^{\mu \nu}\left(\frac{1}{\varepsilon^{\prime}}+5+\frac{2}{\varepsilon}\right),
\end{array}
$$

and the difference between the two regularization schemes is

$$
\Delta(2 d 2)=\Delta(2 d 3)=-C_{F} B_{t}^{\mu \nu} .
$$

One notices that the effect of the wave function renormalization consists of a complete removal of the quark self-energy diagrams with external legs on-shell, as it is required by the second condition (2.8). We can also write the contribution of the quark self-energy with external legs off-shell, graph (2d1), after addition of the mass renormalization counterterm:

$$
\begin{aligned}
M_{(2 \mathrm{~d} 1)}^{\mu \nu} & =C_{F} B_{t}^{\mu \nu}\left(-1 / \varepsilon^{\prime}-t / T+\ln \left(-t / m^{2}\right)\left(4 t / T+t^{2} / T^{2}-4\right)\right) \\
& -i C_{F} T^{b} T^{a} m \gamma^{\mu} \gamma^{\nu}\left(1-2 \ln \left(-t / m^{2}\right)-\ln \left(-t / m^{2}\right) t / T\right) / T .
\end{aligned}
$$

The difference between the DRED and DREG results is

$$
\Delta(2 d 1)=-C_{F} B_{t}^{\mu \nu} .
$$

The remaining quark self-energy diagrams (3i1) and (3i2) with external on-shell legs are derived in analogy to the ones considered above:

$$
\begin{gathered}
M_{(3 \mathrm{i} 1)}^{\mu \nu}=M_{(3 \mathrm{i})}^{\mu \nu}=-C_{F} B_{s}^{\mu \nu}\left(\frac{1}{\varepsilon^{\prime}}+4+\frac{2}{\varepsilon}\right), \\
\Delta(3 i 1)=\Delta(3 i 2)=-C_{F} B_{s}^{\mu \nu} .
\end{gathered}
$$

Concerning the gluon self-energy graphs (2e1) and (2e2) with external legs on-shell, the only nonvanishing contribution they receive from the loop with internal heavy quarks is given by

$$
M_{(2 \mathrm{e} 1)}^{\mu \nu}=M_{(2 \mathrm{e} 2)}^{\mu \nu}=-B_{t}^{\mu \nu} \frac{1}{\varepsilon^{\prime}} \frac{2}{3} .
$$

However, these contributions are explicitly subtracted (together with the logarithmic term $\ln \left(\mu^{2} / m^{2}\right)$ coming from the common factor $C_{\varepsilon}\left(m^{2}\right)$, see eqs. (2.3) and (2.4)) in the on-shell renormalization prescription, in order to avoid the appearance of the large mass logarithms 
from the gluon self-energy diagrams with off-shell external legs in the low energy limit. Therefore, due to the UV counterterm that subtracts that very same loop with heavy quarks, there are no finite contributions to the matrix element from these diagrams. However, at the same time this counterterm introduces the pole terms from the light quark loop sector that are needed to cancel soft and collinear poles from the other parts of the amplitude, e.g. from the real bremsstrahlung part. This indicates that in practice it is very hard to completely disentangle UV and IR/M poles in heavy flavor production and in most cases one obtains a mixture of both instead.

For the reasons specified above it is convenient to present a gauge field renormalization constant $Z_{3}$, used for the gluon self-energy subtraction:

$$
\begin{aligned}
Z_{3} & =1+\frac{g^{2}}{\varepsilon^{\prime}}\left\{\left(\frac{5}{3} N_{C}-\frac{2}{3} n_{l f}\right) C_{\varepsilon}\left(\mu^{2}\right)-\frac{2}{3} C_{\varepsilon}\left(m^{2}\right)\right\} \\
& =1+\frac{g^{2}}{\varepsilon^{\prime}}\left\{\left(b-2 N_{C}\right) C_{\varepsilon}\left(\mu^{2}\right)-\frac{2}{3} C_{\varepsilon}\left(m^{2}\right)\right\},
\end{aligned}
$$

with the QCD beta function $b=\left(11 N_{C}-2 n_{l f}\right) / 6$ containing only light quarks. $N_{C}=3$ is the number of colors.

Similarly to the diagrams (2e1) and (2e2), diagrams (3j1) and (3j2) also vanish due to the explicit decoupling of the heavy quarks in our subtraction prescription. However, instead of doing renormalizations separately for each Feynman diagram, one can chose to employ the renormalization group invariance of the cross section and do only a mass and coupling constant renormalization. In that case, knowing the results for gluon self-energies turns out to be useful in checking the complete cancellation of UV poles by just rescaling the coupling constant in the LO terms $g_{\text {bare }} \rightarrow Z_{g} g$ :

$$
M_{(3 \mathrm{j} 1)}^{\mu \nu}=M_{(3 \mathrm{j} 2)}^{\mu \nu}=-B_{s}^{\mu \nu} \frac{1}{\varepsilon^{\prime}} \frac{2}{3} .
$$

Finally we arrive at the gluon-self energy graph $(3 \mathrm{~h})$, which contains the off-shell gluon self-energy loop that is used for the derivation of the renormalization constant $Z_{3}$. We have evaluated the internal loop in the Feynman gauge. Since it is explicitly gauge invariant, we should arrive at the same result in any other gauge. In our result we show separately the gauge invariant pieces for gluon plus ghost, light quarks and a heavy quark flow inside the loop:

$$
M_{(3 \mathrm{~h})}^{\mu \nu}=B_{s}^{\mu \nu}\left\{\left[N_{C}\left(\frac{1}{\varepsilon^{\prime}} \frac{5}{3}+\frac{31}{9}\right)-n_{l f}\left(\frac{1}{\varepsilon^{\prime}} \frac{2}{3}+\frac{10}{9}\right)\right] \frac{C_{\varepsilon}(-s)}{C_{\varepsilon}\left(m^{2}\right)}-\frac{1}{\varepsilon^{\prime}} \frac{2}{3} I\right\},
$$

with

$$
I=1+\varepsilon^{\prime}\left\{\frac{5}{3}+\frac{4 m^{2}}{s}+\left(1+\frac{2 m^{2}}{s}\right) \beta \ln (x)\right\} .
$$

In (2.24) we have made use of the definitions

$$
\beta \equiv \sqrt{1-4 m^{2} / s}, \quad x \equiv \frac{1-\beta}{1+\beta},
$$


where $\beta$ is the velocity of the heavy quark.

We emphasize that in the last term of $(2.23)$ the $Z_{3}$ counterterm together with the UV pole will remove also the $\ln \left(\mu^{2} / m^{2}\right)$ contribution, while $\ln \left(-\mu^{2} / s\right)$ from the first two terms in (2.23) will be left unsubtracted. Expression (2.23) is obtained for the physical condition $s \geq 4 m^{2}$ for producing a heavy quark pair. However, if one is interested in the case when $s<4 m^{2}$, then in (2.24) one should make the replacement

$$
\beta \ln (x) \rightarrow-2 \sqrt{4 m^{2} / s-1} \arctan \frac{1}{\sqrt{4 m^{2} / s-1}} .
$$

We note that there is a minor problem with preserving gauge invariance when calculating the graph $(3 \mathrm{~h})$ in DRED. It is associated with an $\varepsilon$-dimensional part of one of the $n$ dimensional metric tensors $g_{\mu \nu}^{n}$ that arises in every partonic loop and hampers collecting together similar terms. However, this problem appears to be an artificial one, as in this particular case it makes no difference whether one uses 4 - or $n$-dimensional metric tensor for the evaluation of this gluon self-energy graph. For this reason in practice one would set this $g_{\mu \nu}^{n}$ metric tensor to be the 4-dimensional one. Or, more exactly, if one introduces a proper counterterm so that to restore gauge invariance of the gluon self-energy, then the expression in DRED would be exactly the same as in (2.23), except for the term proportional to $N_{C}$, where one would have $28 / 9$ instead of $31 / 9$, leading to

$$
\Delta(3 h)=-B_{s}^{\mu \nu} .
$$

Concluding our discussion on the 2-point functions we remark that the matrix elements for the additional u-channel 2-point functions can be obtained from eqs. (2.13), (2.16) and (2.20) by the transformation (2.5).

We start by considering the t- and u-channel vertex diagrams. In particular we begin with the purely nonabelian graph $(2 \mathrm{~b})$, which contains a four-point gluon vertex. It is finite, e.g. does not have UV and IR poles. For convenience we define the function

$$
f_{\text {lld }}=4 \operatorname{Li}_{2}(-x)+\ln ^{2}(x)+2 \zeta(2),
$$

with $\zeta(2)=\frac{\pi^{2}}{6}$.

Then the matrix element takes the form

$$
\begin{aligned}
M_{(2 \mathrm{~b})}^{\mu \nu}= & i N_{C}\left\{T ^ { b } T ^ { a } \left[4\left(2 m g^{\mu \nu}-2 p_{3}^{\nu} \gamma^{\mu}-p_{4}^{\nu} \gamma^{\mu}+p_{3}^{\mu} \gamma^{\nu}+2 p_{4}^{\mu} \gamma^{\nu}\right)\left(s \beta \ln \left(s / m^{2}\right)-m^{2} f_{\text {lld }}\right)-\right.\right. \\
& \left.\left.3 m s \gamma^{\mu} \gamma^{\nu}\left(4 \beta \ln \left(s / m^{2}\right)-f_{\text {lld }}\right)\right] / 4 s^{2} \beta^{3}+(a \leftrightarrow b, \mu \leftrightarrow \nu)\right\}- \\
& i \delta^{a b}\left[2\left(2 m g^{\mu \nu}+p_{3}^{\nu} \gamma^{\mu}-p_{4}^{\nu} \gamma^{\mu}+p_{3}^{\mu} \gamma^{\nu}-p_{4}^{\mu} \gamma^{\nu}\right)\left(s \beta \ln \left(s / m^{2}\right)-m^{2} f_{\text {lld }}\right)-\right. \\
& \left.3 m s \beta^{2} g^{\mu \nu} f_{\text {lld }}\right] / 4 s^{2} \beta^{3} .
\end{aligned}
$$

It is easily seen from eq. (2.29) that graph $(2 \mathrm{~b})$ is explicitly t-u symmetric, as it follows from its geometric topology. Because $M_{(2 \mathrm{~b})}^{\mu \nu}$ does not exibit any UV poles, there is no difference between DREG and DRED in this case, e.g.

$$
\Delta(2 b)=0 .
$$


Next we turn to graphs (2c1) and (2c2) and define another useful function:

$$
f_{\mathrm{dl}}=\zeta(2)-\mathrm{Li}_{2}\left(\frac{T}{m^{2}}\right) .
$$

Diagrams of this topology do not only occur in hadroproduction, but also in other processes such as photoproduction and $\gamma \gamma$ production of heavy flavors. For this reason we also present the corresponding t-channel color factors for these graphs. Then it is straightforward to separate our Dirac structure from the color coefficients and one can easily deduce results for other processes where these graphs contribute, though with different color weights. The color factor for both (2c1) and (2c2) diagrams is the same:

$$
T_{\mathrm{col}}^{(2 \mathrm{c} 1)}=T_{\mathrm{col}}^{(2 \mathrm{c} 2)}=\left(C_{F}-\frac{N_{C}}{2}\right) T^{b} T^{a}=-\frac{1}{6} T^{b} T^{a} .
$$

The complete matrix elements are:

$$
\begin{aligned}
M_{(2 \mathrm{c} 1)}^{\mu \nu} & =B_{t}^{\mu \nu}\left[-1 / \varepsilon^{\prime}+2 f_{\mathrm{dl}} m^{2} / t-\ln \left(-t / m^{2}\right)\left(6 m^{2}+t\right) / T\right] / 6 \\
& +i T^{b} T^{a}\left\{p_{3}^{\mu} \gamma^{\nu}\left[2 f_{\mathrm{dl}} m^{2} T / t^{2}+\ln \left(-t / m^{2}\right)\left(m^{2} / T-2 T / t\right)+1\right]\right. \\
& \left.-m p_{3}^{\mu} \not p_{1} \gamma^{\nu}\left[\ln \left(-t / m^{2}\right) / T-1 / t\right]+m \gamma^{\mu} \gamma^{\nu} \ln \left(-t / m^{2}\right)\right\} / 3 T .
\end{aligned}
$$

Because one has a UV pole $1 / \varepsilon^{\prime}$ (the prime denotes UV poles), there is a finite difference between the two n-dimensional schemes:

$$
\Delta(2 c 1)=\left(C_{F}-\frac{N_{C}}{2}\right) B_{t}^{\mu \nu} .
$$

As expected, the difference is proportional to the sum of the Born terms of the relevant production channels. We emphasize that one has to take care when calculating matrix elements in DRED. In particular, one has to keep a clear distinction between the 4-dimensional and the $n$-dimensional metric tensors $g^{\mu \nu}$ when doing their convolutions. Failing to do so may very well result in introducing extra terms that do not satisfy the Slavnov-Taylor identities [20].

For the graph $(2 \mathrm{c} 2)$ we obtain:

$$
\begin{aligned}
M_{(2 \mathrm{c} 2)}^{\mu \nu} & =B_{t}^{\mu \nu}\left[-1 / \varepsilon^{\prime}+2 f_{\mathrm{dl}} m^{2} / t-\ln \left(-t / m^{2}\right)\left(6 m^{2}+t\right) / T\right] / 6 \\
& +i T^{b} T^{a}\left\{p_{4}^{\nu} \gamma^{\mu}\left[-2 f_{\mathrm{dl}} m^{2} T / t^{2}+\ln \left(-t / m^{2}\right)\left(m^{2} / T+2 T / t\right)-2 m^{2} / t-1\right]\right. \\
& +m p_{4}^{\nu} \gamma^{\mu} \phi_{1}\left[\ln \left(-t / m^{2}\right) / T-1 / t\right]+m \gamma^{\mu} \gamma^{\nu} \ln \left(-t / m^{2}\right) \\
& \left.-2 m p_{3}^{\mu} p_{4}^{\nu}\left[\ln \left(-t / m^{2}\right) / T-1 / t\right]\right\} / 3 T
\end{aligned}
$$

and

$$
\Delta(2 c 2)=\left(C_{F}-\frac{N_{C}}{2}\right) B_{t}^{\mu \nu} .
$$

To write down the results for graphs (2c3) and (2c4) we introduce one more function:

$$
f_{\mathrm{ll}}=\ln ^{2}\left(-\frac{t}{m^{2}}\right)+\mathrm{Li}_{2}\left(\frac{T}{m^{2}}\right) .
$$


The color factors for both diagrams are the same:

$$
T_{\mathrm{col}}^{(2 \mathrm{c} 3)}=T_{\mathrm{col}}^{(2 \mathrm{c} 4)}=-\frac{N_{C}}{2} T^{b} T^{a}=-\frac{3}{2} T^{b} T^{a} .
$$

Graphs (2c3) and (2c4) exhibit a rich structure of UV and IR singularities. Here one of the scalar integrals that is needed for the reduction of tensor integrals is of the type

$$
\int \frac{d^{n} k}{k^{2}(k+p)^{2}}
$$

which is effectively zero in dimensional regularization. However, we choose to separate UV and IR/M poles to keep trace of all the sources of UV singularities, e.g. the above mentioned integral is proportional to the difference $1 / \varepsilon^{\prime}-1 / \varepsilon$. This is our procedure for the relevant vertex diagrams. Thus,

$$
\begin{aligned}
M_{(2 \mathrm{c} 3)}^{\mu \nu} & =3 B_{t}^{\mu \nu}\left[3 / \varepsilon^{\prime}-1 / \varepsilon^{2}+\left(2 \ln \left(-t / m^{2}\right)-1\right) / \varepsilon+4+6 \ln \left(-t / m^{2}\right) m^{2} / T-2 f_{11}\right] / 2 \\
& +3 i T^{b} T^{a}\left\{p _ { 3 } ^ { \mu } \gamma ^ { \nu } \left[-1 / \varepsilon^{2}+2 / \varepsilon+2 \ln \left(-t / m^{2}\right) / \varepsilon-2 f_{\mathrm{ll}}+2 \ln \left(-t / m^{2}\right)\right.\right. \\
& \left.\times m^{2}\left(m^{2} / T+1\right) / T+2 m^{2} / T+6\right] / 2 t-3 m \gamma^{\mu} \gamma^{\nu} \ln \left(-t / m^{2}\right) / 2 T \\
& \left.-m p_{3}^{\mu} \not p_{1} \gamma^{\nu}\left[\ln \left(-t / m^{2}\right)\left(m^{2}+T\right)+T\right] / t T^{2}\right\}
\end{aligned}
$$

with

$$
\Delta(2 c 3)=\frac{N_{C}}{2} B_{t}^{\mu \nu}
$$

And

$$
\begin{aligned}
M_{(2 \mathrm{c} 4)}^{\mu \nu} & =3 B_{t}^{\mu \nu}\left[3 / \varepsilon^{\prime}-1 / \varepsilon^{2}+\left(2 \ln \left(-t / m^{2}\right)-1\right) / \varepsilon+4+6 \ln \left(-t / m^{2}\right) m^{2} / T-2 f_{11}\right] / 2 \\
& +3 i T^{b} T^{a}\left\{p _ { 4 } ^ { \nu } \gamma ^ { \mu } \left[1 / \varepsilon^{2}-2 / \varepsilon-2 \ln \left(-t / m^{2}\right) / \varepsilon+2 f_{11}+2 \ln \left(-t / m^{2}\right)\right.\right. \\
& \left.\times m^{2}\left(m^{2} / T+1\right) / T+2 m^{2} / T-6\right] / 2 t-3 m \gamma^{\mu} \gamma^{\nu} \ln \left(-t / m^{2}\right) / 2 T \\
& \left.+m p_{4}^{\nu} \gamma^{\mu} p_{1}\left[\ln \left(-t / m^{2}\right)\left(m^{2}+T\right)+T\right] / t T^{2}-2 m p_{3}^{\mu} p_{4}^{\nu}\left[\ln \left(-t / m^{2}\right)\left(m^{2}+T\right)+T\right] / t T^{2}\right\}
\end{aligned}
$$

with

$$
\Delta(2 c 4)=\frac{N_{C}}{2} B_{t}^{\mu \nu}
$$

The results for the matrix elements of the additional u-channel vertex graphs are obtained from eqs. $(2.33),(2.35),(2.40)$ and $(2.42)$ by the transformation (2.5). However, there is a subtle point involved here: we stress that for the graphs $(2 \mathrm{c} 3)$ and $(2 \mathrm{c} 4)$ transformation (2.5) transforms the t-channel result of the graph $(2 \mathrm{c} 3)$ to the $\mathrm{u}$-channel result for the graph $(2 \mathrm{c} 4)$, while the t-channel result of $(2 \mathrm{c} 4)$ goes to the $\mathrm{u}$-channel result for $(2 \mathrm{c} 3)$. This is important to keep in mind when dealing with reactions which involve asymmetric set of graphs as e.g. photoproduction of heavy flavors.

It is worthwhile to mention that we have tested the above results for the $\mathrm{t}$ - and $\mathrm{u}$ channel contributions against the ones given in [12] and [15] by folding our NLO matrix 
elements with the Born term matrix elements for the cases of unpolarized and longitudinally polarized incoming bosons. We obtain full agreement.

Next we turn to the remaining s-channel graphs shown in Fig. 3. For all the gluon propagators we work in Feynman gauge. Although this set of graphs is purely nonabelian for QCD type one-loop corrections, there could be also abelian (e.g. QED) virtual corrections to graph (3f1). For this reason we also give the color factor for it separately:

$$
T_{\mathrm{col}}^{(3 \mathrm{f} 1)}=\left(C_{F}-\frac{N_{C}}{2}\right)\left(T^{a} T^{b}-T^{b} T^{a}\right)=-\frac{1}{6}\left(T^{a} T^{b}-T^{b} T^{a}\right) .
$$

In the result for this graph together with the UV divergence we clearly see a collinear pole as well, multiplied by a logarithmic factor, that should be cancelled against the corresponding term in the factorization counterterm of the real bremsstrahlung:

$$
\begin{gathered}
M_{(3 \mathrm{f} 1)}^{\mu \nu}=\left\{-B_{s}^{\mu \nu} s\left[s \beta / \varepsilon^{\prime}+\left(2 m^{2}-s\right)\left(2 \ln (x) / \varepsilon-4 \operatorname{Li}_{2}(x)-4 \ln (x) \ln (1-x)+6 \ln (x)\right.\right.\right. \\
\left.\left.+\ln ^{2}(x)-8 \zeta(2)\right)+3 \ln (x) s\right] \\
\left.+2 i\left(T^{a} T^{b}-T^{b} T^{a}\right) m \ln (x)\left[-g^{\mu \nu}(s+2 t)-4 p_{3}^{\mu} p_{4}^{\nu}+4 p_{4}^{\mu} p_{3}^{\nu}\right]\right\} / 6 s^{2} \beta \\
\Delta_{(3 \mathrm{f} 1)}=\left(C_{F}-\frac{N_{C}}{2}\right) B_{s}^{\mu \nu} .
\end{gathered}
$$

Graph (3f2) contributes as:

$$
\begin{aligned}
M_{(3 \mathrm{f} 2)}^{\mu \nu} & =N_{C}\left\{B_{s}^{\mu \nu}\left[3 s \beta^{2}\left(1 / \varepsilon^{\prime}+2\right)-\ln \left(s / m^{2}\right)\left(8 m^{2}-s\right)+f_{\mathrm{lld}} m^{2} / \beta\right]\right. \\
& +2 i\left(T^{a} T^{b}-T^{b} T^{a}\right) m\left[g^{\mu \nu}(s+2 t)+4 p_{3}^{\mu} p_{4}^{\nu}-4 p_{4}^{\mu} p_{3}^{\nu}\right] \\
& \left.\times\left[\ln \left(s / m^{2}\right)\left(8 m^{2}+s\right) / \beta^{2}-2 s-3 f_{\mathrm{lld}} m^{2} / \beta^{3}\right] / s^{2}\right\} / 2 s \beta^{2} .
\end{aligned}
$$

The difference between the two regularization schemes is again determined by the coefficient of the UV pole:

$$
\Delta_{(3 \mathrm{f} 2)}=\frac{N_{C}}{2} B_{s}^{\mu \nu}
$$

We finish our consideration of the vertex diagrams for gluon fusion with the triangle graph contribution (tri) $\equiv(3 g 1)+(3 g 2)$, e.g. we sum the two graphs $(3 g 1)$ and $(3 g 2)$. For the case when one has gluons and ghosts inside the triangle loop we obtain:

$$
\begin{aligned}
M_{\text {(tri) }}^{\mu \nu}(g) & =N_{C}\left\{B _ { s } ^ { \mu \nu } \left[33 / \varepsilon^{\prime}-36 / \varepsilon^{2}-171 / \varepsilon+36 \ln \left(s / m^{2}\right) / \varepsilon+138 \ln \left(s / m^{2}\right)-18 \ln ^{2}\left(s / m^{2}\right)\right.\right. \\
& +144 \zeta(2)-284]+6 i\left(T^{a} T^{b}-T^{b} T^{a}\right) \not p_{1}\left[g ^ { \mu \nu } \left(27 / \varepsilon^{\prime}-6 / \varepsilon^{2}-33 / \varepsilon+6 \ln \left(s / m^{2}\right) / \varepsilon\right.\right. \\
& \left.\left.\left.+6 \ln \left(s / m^{2}\right)-3 \ln ^{2}\left(s / m^{2}\right)+24 \zeta(2)-4\right) / s-p_{2}^{\mu} p_{1}^{\nu} 16 / s^{2}\right]\right\} / 72 ;
\end{aligned}
$$

For the two more cases when one has light and heavy quarks inside the loop we get

$$
M_{\text {(tri) }}^{\mu \nu}(q)=2 n_{l f}\left\{B_{s}^{\mu \nu}\left[3 / \varepsilon^{\prime}-3 \ln \left(s / m^{2}\right)+5\right]-3 i\left(T^{a} T^{b}-T^{b} T^{a}\right) p_{1}\left[g^{\mu \nu} / s-2 p_{2}^{\mu} p_{1}^{\nu} / s^{2}\right]\right\} / 9
$$


with $n_{l f}$ number of light flavors in the triangle loop, while for the heavy flavor case one has

$$
\begin{aligned}
M_{(\mathrm{tri})}^{\mu \nu}(Q) & =2\left\{B_{s}^{\mu \nu}\left[3 / \varepsilon^{\prime}+3 \ln (x)\left(2 m^{2} / s+1\right) \beta+5+12 m^{2} / s\right]\right. \\
& -3 i\left(T^{a} T^{b}-T^{b} T^{a}\right) p_{1}\left[g^{\mu \nu} / s^{2}-2 p_{2}^{\mu} p_{1}^{\nu} / s^{3}\right]\left[3(\ln (x)+4 \beta) \ln (x) m^{2}\right. \\
& \left.\left.-18 \zeta(2) m^{2}+24 m^{2}+s\right]\right\} / 9 .
\end{aligned}
$$

The complete matrix element for the triangle is the sum of the above three expressions (2.49), (2.50) and (2.51):

$$
M_{(\mathrm{tri})}^{\mu \nu}=M_{(\mathrm{tri})}^{\mu \nu}(g)+M_{(\mathrm{tri})}^{\mu \nu}(q)+M_{(\mathrm{tri})}^{\mu \nu}(Q) .
$$

We have compared our results in eqs. (2.49) and (2.50) with those available in the literature [21], and found agreement.

The difference between dimensional reduction and regularization arises due to gluons in the loop:

$$
\Delta_{(\operatorname{tri})}=B_{s}^{\mu \nu}
$$

\section{RESULTS FOR THE BOX DIAGRAMS IN GLUON FUSION}

In this chapter we describe the technically most complicated derivation of the 4-point massive loop diagrams. The results of this chapter cannot be deduced from the results of any other relevant publications up to date. The four box graphs (2a1)-(2a4) contributing to the subprocess $g+g \rightarrow Q+\bar{Q}$ are depicted in Fig. 2. We have used an adapted version of the Passarino-Veltman techniques [22] to reduce tensor integrals to scalar ones. The scalar integrals are taken from [5] which have been checked by us and in [8].

First of all we note that the results for the box diagrams are the same both in DREG and DRED, as a consequence of the ultraviolet convergence of the box graphs.

We expand all the box diagrams arising at the one loop level in gluon fusion in terms of eight independent Dirac structures. In turn, coefficients of the Dirac structures are expanded in terms of universal independent Lorentz objects. And finally, the coefficients of the Lorentz structures are expanded as products of a small set of analytic functions and various coefficient functions, which are combinations of scalar Mandelstam variables of the subprocess under consideration. Note that the Dirac structures and Lorentz objects are the same for every box diagram. In particular, we cast the box matrix element into the following universal form:

$$
\begin{aligned}
M^{\mu \nu} & =i T_{\mathrm{col}}\left\{M_{\mathrm{Bt}}^{\mu \nu} \sum f_{i} b_{i}\right. \\
& +\not p_{1}\left[g^{\mu \nu} \sum f_{i} b_{i 1}^{(p)}+p_{3}^{\mu} p_{3}^{\nu} \sum f_{i} b_{i 2}^{(p)}+p_{3}^{\mu} p_{4}^{\nu} \sum f_{i} b_{i 3}^{(p)}+p_{4}^{\mu} p_{3}^{\nu} \sum f_{i} b_{i 4}^{(p)}+p_{4}^{\mu} p_{4}^{\nu} \sum f_{i} b_{i 5}^{(p)}\right] \\
& +\gamma^{\mu}\left[p_{3}^{\nu} \sum f_{i} b_{i 1}^{(m)}+p_{4}^{\nu} \sum f_{i} b_{i 2}^{(m)}\right]+\gamma^{\nu}\left[p_{3}^{\mu} \sum f_{i} b_{i 1}^{(n)}+p_{4}^{\mu} \sum f_{i} b_{i 2}^{(n)}\right]+m \gamma^{\mu} \gamma^{\nu} \sum f_{i} c_{i}
\end{aligned}
$$




$$
\begin{aligned}
& +m \gamma^{\mu} \not p_{1}\left[p_{3}^{\nu} \sum f_{i} d_{i 1}+p_{4}^{\nu} \sum f_{i} d_{i 2}\right]+m \gamma^{\nu} \not p_{1}\left[p_{3}^{\mu} \sum f_{i} e_{i 1}+p_{4}^{\mu} \sum f_{i} e_{i 2}\right] \\
& \left.+m\left[g^{\mu \nu} \sum f_{i} g_{i 1}+p_{3}^{\mu} p_{3}^{\nu} \sum f_{i} g_{i 2}+p_{3}^{\mu} p_{4}^{\nu} \sum f_{i} g_{i 3}+p_{4}^{\mu} p_{3}^{\nu} \sum f_{i} g_{i 4}+p_{4}^{\mu} p_{4}^{\nu} \sum f_{i} g_{i 5}\right]\right\} \\
& +\mathcal{M}_{t} \leftrightarrow \mathcal{M}_{u} .
\end{aligned}
$$

Even though the number of independent covariants for the process $g+g \rightarrow Q+\bar{Q}$ is eight for $n=4$ this will not be the case for $n \neq 4$ relevant to this application. We have therefore made no attempt to reduce the above number of covariants to a minimal set.

Depending on the type of the box graph one has different number of terms under the summation signs in (3.1). These numbers as well as the analytic functions $f_{i}$ are specified below. The coefficient functions $b_{i}, \ldots, g_{i 5}$ are given in Appendix A of this paper.

The t-channel Born term matrix structure $M_{\mathrm{Bt}}^{\mu \nu}$ is the same for all box graphs and is defined as

$$
M_{\mathrm{Bt}}^{\mu \nu} \equiv \gamma^{\mu}\left(\hat{p}_{3}-\hat{p}_{1}+m\right) \gamma^{\nu},
$$

which, when taken between the spin wave functions implying the effective relations $p_{1}^{\mu}=$ $0, p_{2}^{\nu}=0$, can be written as

$$
M_{\mathrm{Bt}}^{\mu \nu}=2 p_{3}^{\mu} \gamma^{\nu}-\gamma^{\mu} \not p_{1} \gamma^{\nu}
$$

Furthermore, for the box diagrams (2a1) and (2a2) we empirically found the following relations between the $d$ and $e$ coefficients:

$$
e_{i 1}=d_{i 2}, \quad e_{i 2}=d_{i 1}
$$

For the 4-point graph (2a4) the above relations are slightly changed:

$$
\begin{aligned}
e_{i 1} & =d_{i 2}, \quad e_{i 2}=d_{i 1}, \quad \text { for } \quad i=1,2,4,5,8,9 ; \\
e_{31} & =d_{32}+2 m^{2} u /\left(m^{2} s-u t\right)^{2}, \quad e_{61}=2 d_{62}, \quad e_{71}=2 d_{72}, \\
e_{32} & =d_{31}-2 m^{2} t /\left(m^{2} s-u t\right)^{2}, \quad e_{62}=2 d_{61}, \quad e_{72}=2 d_{71} .
\end{aligned}
$$

Because of the relations (3.4) and (3.5), we will not write down the results for the $e$ coefficients in the Appendix A.

We note that for the boxes (2a3) and (2a4) the $\mathcal{M}_{t} \leftrightarrow \mathcal{M}_{u}$ term in (3.1) is absent as there does not exist a u-channel diagram for them. Furthermore, it is easy to see that boxes (2a3) and (2a4) go into each other with $\mathcal{M}_{t} \leftrightarrow \mathcal{M}_{u}$. For this reason we write down explicit results only for one of these boxes in App. A.

Next we present the color factors and functions for the abelian type box diagram (2a1). For this graph the sums over $i$ in (3.1) run from 1 to 7 for all the terms except for the term that multiplies $M_{\mathrm{Bt}}^{\mu \nu}$, which runs from 1 to 8 . We have:

$$
T_{\mathrm{col}}=\frac{1}{4} \delta^{a b}+\left(C_{F}-\frac{N_{C}}{2}\right) T^{b} T^{a}
$$




$$
\begin{aligned}
& f_{1}=\ln (x), \quad f_{2}=\ln ^{2}(x), \\
& f_{3}=-2 \ln (x) \ln (1+x)+2 \ln (x) \ln \left(-\frac{t}{m^{2}}\right)-2 \operatorname{Li}_{2}(-x)+3 \zeta(2), \quad f_{4}=\mathrm{f}_{\mathrm{dl}}, \\
& f_{5}=\zeta(2), \quad f_{6}=\ln \left(-\frac{t}{m^{2}}\right), \quad f_{7}=1, \quad f_{8}=\ln (x) \ln (1-x)+\operatorname{Li}_{2}(x) .
\end{aligned}
$$

For the nonabelian box diagram (2a2) the sums over $i$ in (3.1) run from 1 to 8 for all terms except for the one that multiplies $M_{\mathrm{Bt}}^{\mu \nu}$, which runs from 1 to 9 . For the color factor we obtain:

$$
T_{\mathrm{col}}=\frac{1}{4} \delta^{a b}+\frac{N_{C}}{2} T^{b} T^{a}
$$

The relevant nine analytic functions that describe the result of evaluating the box diagram (2a2) are given by

$$
\begin{aligned}
f_{1} & =\ln \left(\frac{s}{m^{2}}\right), \quad f_{2}=\ln ^{2}\left(\frac{s}{m^{2}}\right), \quad f_{3}=\ln ^{2}\left(\frac{s}{m^{2}}\right)+4 \mathrm{f}_{l 1}-4 \ln \left(\frac{s}{m^{2}}\right) \ln \left(-\frac{t}{m^{2}}\right)+2 \zeta(2) \\
f_{4} & =\mathrm{f}_{\mathrm{lld}}, \quad f_{5}=\zeta(2), \quad f_{6}=\ln \left(-\frac{t}{m^{2}}\right), \quad f_{7}=1, \quad f_{8}=\mathrm{f}_{\mathrm{ll}} \\
f_{9} & =\ln \left(\frac{s}{m^{2}}\right) \ln \left(-\frac{t}{m^{2}}\right) .
\end{aligned}
$$

In the case of the crossed box (2a4) one has nine terms for every sum in (3.1). The color factor for this graph takes the simple form

$$
T_{\mathrm{col}}=\frac{1}{4} \delta^{a b} .
$$

We found it convenient to define functions $f_{i}$ as follows:

$$
\begin{aligned}
& f_{1}=\mathrm{f}_{\mathrm{ll}}, \quad f_{2}=\mathrm{f}_{\mathrm{ll}}(t \rightarrow u), \\
& f_{3}=\mathrm{f}_{\mathrm{dl}}+\mathrm{f}_{\mathrm{dl}}(t \rightarrow u)-\mathrm{f}_{\mathrm{ll}}-\mathrm{f}_{\mathrm{ll}}(t \rightarrow u)+2 \ln \left(-\frac{t}{m^{2}}\right) \ln \left(-\frac{u}{m^{2}}\right)-4 \zeta(2), \\
& f_{4}=\mathrm{f}_{\mathrm{dl}}, \quad f_{5}=\zeta(2), \quad f_{6}=\ln \left(-\frac{t}{m^{2}}\right), \quad f_{7}=\ln \left(-\frac{u}{m^{2}}\right), \\
& f_{8}=\ln \left(-\frac{t}{m^{2}}\right) \ln \left(-\frac{u}{m^{2}}\right), \quad f_{9}=1 .
\end{aligned}
$$

In order to compare with existing results we have folded the one-loop contribution with the LO Born term results. Using our results for the box matrix elements we have reproduced all the unpolarized and polarized amplitudes presented in the papers [12] and [15], where the Born term was folded in at the very beginning of the calculation. Note that after folding with the LO Born term many powers in the numerators and denominators cancel out, leading to a very short expressions for the box amplitudes.

However, the above checks and the ones mentioned in the Section II did not include all the graphs for the gluon fusion subprocess. For a further check on the correctness of 
the results for all of our matrix elements in Figs. 2 and 3 we have performed a global check on the gauge invariance by first excluding one of the heavy quark momenta from the independent set of momenta (see Ref. [19] for details), e.g. reexpressing $p_{4}^{\nu}$ in terms of $p_{1}^{\nu}$ and $p_{3}^{\nu}$, then contracting the matrix elements by $p_{1}^{\mu}$. As well, we have performed the second possible check, contracting our matrix elements by the momentum of the other massless boson $p_{2}^{\nu}$. We have verified gauge invariance for the following gauge-invariant subsets of diagrams: (i) When the incoming gauge bosons are photons, i.e. including graphs (2a1), (2c1), (2c2), (2d1), (2d2), (2d3) and their u-channel counterparts, with corresponding color weights; (ii) For the photoproduction of heavy flavors, i.e. including all the above diagrams plus graphs (2a4), (2c4), (2e1) and their u-channel counterparts, with corresponding color weights; (iii) For the hadroproduction of heavy flavors, which ultimately includes all the graphs from Figs. 2 and 3 and their relevant $\mathrm{u}$-channel counterparts. We emphasize that the above gauge invariance checks were made separately for both color structures $C_{F}$ and $N_{C}$, and for every existing combination of color matrices $T^{a}, T^{b}$ and $\delta^{a b}$, whenever they arise. Also, for the hadroproduction set of graphs we had to set $\varepsilon^{\prime}=\varepsilon$ as there is a mixing of UV and IR poles in the s-channel, particularly for the triangle graph (3g1) with gluons in the loop.

At the end of our discussion for the gluon fusion subprocess we comment on the DRED result. First we note that at the one-loop level the $Z_{1 F}$ vertex renormalization constant is a sum of a wave function renormalization constant $Z_{2}$, which is equal to the abelian vertex renormalization constant $Z_{1 V}$, and the term that is proportional to the $N_{C}$, which does not differ in DREG and DRED:

$$
Z_{1 F}=Z_{2}-\frac{g^{2}}{\varepsilon} C_{\varepsilon}\left(\mu^{2}\right) N_{C}
$$

Most importantly, the modification of the renormalization constants $Z_{2}$ and $Z_{1 F}$ in DRED does not affect the other renormalization constants and preserves the form of the SlavnovTaylor identities for all those constants by construction.

Next we take the set of graphs relevant to heavy flavor production in photon-photon collisions which are the set of graphs mentioned in item (i) above. For this abelian set of graphs the overall action of the renormalization counterterms amounts to completely removing the quark self-energy graphs with on-shell legs and adding just one term that is equal to one of the removed graphs, e.g. expression (2.13) in DREG or (2.14) in DRED. But this is effectively equivalent to multiplying each of the two self-energy graphs by a factor of $1 / 2$, and leaving out renormalization. Then, correspondingly, the differences between the two dimensional schemes for these self-energy graphs have to be halved, and simple counting will now show that the sum of $\Delta$ 's for this set of graph vanishes identically. Taking into account the above remark on the $Z_{1 F}$ constant, e.g. separating the $C_{F}$ and $N_{C}$ parts of the vertex diagrams during the renormalization procedure, one can see that the same statements apply to the photoproduction set of graphs given by the gauge-invariant set (ii) above. And the same is true also for all the self-energy and vertex graphs for the hadroproduction set. The remaining difference coming from the gluonic loops in graphs 
(3g1) and (3h) cancel. This means that there is no difference between the DREG and DRED virtual NLO corrections for the gauge boson fusion subprocesses.

Finally we note that the original computer output for the box diagrams was extremely long. The final results were cast into the above shorter form with the help of the REDUCE Computer Algebra System [23].

\section{ANNIHILATION OF THE QUARK-ANTIQUARK PAIR}

The graphs contributing to this subprocess are shown in Fig. 4 for the leading order term and in Fig. 5 for the one-loop corrections. The leading order contribution proceeds only through the s-channel graph. One has:

$$
B_{q \bar{q}}=i T^{a} T^{a} \bar{v}\left(p_{2}\right) \gamma^{\mu} u\left(p_{1}\right) \bar{u}\left(p_{3}\right) \gamma_{\mu} v\left(p_{4}\right) / s .
$$

Here the color matrices $T^{a}$ belong to different fermion lines that are connected by the gluon having color index $a$. We have again left out the factor $g^{2}$ in the above equation. In the Passarino-Veltman reduction for tensor integrals we use the same scalar integrals as those appearing in the gluon fusion subprocess, with relevant shifts and interchanges of momenta as needed.

Starting again with the 2-point functions, we notice that the result for graph $(5 \mathrm{~g})$ can be obtained from the one of (2.23) for graph (2h) in the gluon fusion subprocess by the simple replacement

$$
M_{(5 g)}=M_{(2 h)}^{\mu \nu}\left(B_{s}^{\mu \nu} \rightarrow B_{q \bar{q}}\right),
$$

and all the statements after (2.23) are equally applicable to $M_{(5 g)}$.

The massless quark self-energy graphs $(5 \mathrm{j})$ and $(5 \mathrm{k})$ with external legs on-shell vanish identically:

$$
M_{(5 j)}=M_{(5 k)}=0 .
$$

The massive quark self-energy graphs (5h) and (5i) with external legs on-shell are derived analogously to the ones considered in the previous section:

$$
M_{(5 \mathrm{~h})}=M_{(5 \mathrm{i})}=-C_{F} B_{q \bar{q}}\left(\frac{1}{\varepsilon^{\prime}}+4+\frac{2}{\varepsilon}\right),
$$

and the difference between the two regularizations schemes is

$$
\Delta(5 h)=\Delta(5 i)=-C_{F} B_{q \bar{q}}
$$

Results for the vertex diagrams are relatively short. Starting with graphs (5c) and (5d) one finds that they are proportional to the LO Born term:

$$
M_{(5 c)}=B_{q \bar{q}}\left[-1 / \varepsilon^{\prime}+2 / \varepsilon^{2}+4 / \varepsilon-2 \ln \left(s / m^{2}\right) / \varepsilon+\ln ^{2}\left(s / m^{2}\right)-3 \ln \left(s / m^{2}\right)-8 \zeta(2)+8\right] / 6
$$


and

$$
M_{(5 d)}=3 B_{q \bar{q}}\left[3 / \varepsilon^{\prime}-4 / \varepsilon+\ln \left(s / m^{2}\right)-2\right] / 2 .
$$

Corresponding differences between DRED and DREG results are

$$
\Delta(5 c)=\left(C_{F}-\frac{N_{C}}{2}\right) B_{q \bar{q}}, \quad \Delta(5 d)=\frac{N_{C}}{2} B_{q \bar{q}} .
$$

For the other two vertex diagrams we also obtain simple expressions:

$$
\begin{aligned}
M_{(5 e)} & =\left\{B _ { q \overline { q } } \left[-1 / \varepsilon^{\prime}+3 \ln (x) \beta+(1 / \beta+\beta)\left(\ln (x) / \varepsilon-2 \operatorname{Li}_{2}(x)-2 \ln (x) \ln (1-x)\right.\right.\right. \\
& \left.\left.\left.+\ln ^{2}(x) / 2-4 \zeta(2)\right)\right]+4 i T^{a} T^{a} m \bar{v}\left(p_{2}\right) \not p_{3} u\left(p_{1}\right) \bar{u}\left(p_{3}\right) v\left(p_{4}\right) \ln (x) / s^{2} \beta\right\} / 6
\end{aligned}
$$

and

$$
\begin{aligned}
M_{(5 f)} & =3\left\{B_{q \bar{q}}\left[3\left(1 / \varepsilon^{\prime}+2\right)-\ln \left(s / m^{2}\right)\left(8 m^{2} / s-1\right) / \beta^{2}+\mathrm{f}_{\text {lld }} m^{2} / s \beta^{3}\right]-4 i T^{a} T^{a} m(4.10)\right. \\
& \left.\times \bar{v}\left(p_{2}\right) p_{3} u\left(p_{1}\right) \bar{u}\left(p_{3}\right) v\left(p_{4}\right)\left[\ln \left(s / m^{2}\right)\left(8 m^{2} / s+1\right) / \beta^{2}-2-3 \mathrm{f}_{l \mathrm{ld}} m^{2} / s \beta^{3}\right] / s^{2} \beta^{2}\right\} / 2
\end{aligned}
$$

with the function $f_{l l d}$ defined in (2.28). Once again, the differences between the two regularization schemes are

$$
\Delta(5 e)=\left(C_{F}-\frac{N_{C}}{2}\right) B_{q \bar{q}}, \quad \Delta(5 f)=\frac{N_{C}}{2} B_{q \bar{q}}
$$

Turning to the two box diagrams we again note that because of the absence of UV poles there are no differences between DRED and DREG. Extensive Dirac algebra manipulations lead to rather compact expressions for the matrix elements.

Since for the $q \bar{q} \rightarrow Q \bar{Q}$ subprocess one has two spinor "sandwiches" we cannot have momenta with Lorentz indices, and consequently there is no expansion of matrix elements in terms of Lorentz objects. We expanded the box diagrams in terms of the seven independent Dirac structures, the same set for each of the two box graphs. Then every Dirac structure is multiplied by the sums of products of a small set of analytic functions and coefficient functions. Thus, we have the following compact expansion for both box diagrams:

$$
\begin{aligned}
M & =i T_{\text {col }}\left\{\bar{v}\left(p_{2}\right) \gamma^{\mu} u\left(p_{1}\right) \bar{u}\left(p_{3}\right) \gamma_{\mu} v\left(p_{4}\right) \sum f_{i} h_{i}\right. \\
& +\bar{v}\left(p_{2}\right) \not p_{3} u\left(p_{1}\right) \bar{u}\left(p_{3}\right) \not p_{1} v\left(p_{4}\right) \sum f_{i} h_{i}^{(1)} \\
& +\bar{v}\left(p_{2}\right) \gamma^{\nu} \not p_{3} \gamma^{\mu} u\left(p_{1}\right) \bar{u}\left(p_{3}\right) \gamma_{\mu} \not p_{1} \gamma_{\nu} v\left(p_{4}\right) \sum f_{i} h_{i}^{(2)} \\
& +\bar{v}\left(p_{2}\right) \gamma^{\nu} \gamma^{\alpha} \gamma^{\mu} u\left(p_{1}\right) \bar{u}\left(p_{3}\right) \gamma_{\mu} \gamma_{\alpha} \gamma_{\nu} v\left(p_{4}\right) \sum f_{i} h_{i}^{(3)} \\
& +m \bar{v}\left(p_{2}\right) \not p_{3} u\left(p_{1}\right) \bar{u}\left(p_{3}\right) v\left(p_{4}\right) \sum f_{i} h_{i}^{(4)} \\
& +m \bar{v}\left(p_{2}\right) \gamma^{\mu} u\left(p_{1}\right) \bar{u}\left(p_{3}\right) \gamma_{\mu} \not p_{1} v\left(p_{4}\right) \sum f_{i} h_{i}^{(5)} \\
& \left.+m \bar{v}\left(p_{2}\right) \gamma^{\nu} \not p_{3} \gamma^{\mu} u\left(p_{1}\right) \bar{u}\left(p_{3}\right) \gamma_{\mu} \gamma_{\nu} v\left(p_{4}\right) \sum f_{i} h_{i}^{(6)}\right\} .
\end{aligned}
$$

Note that the number of independent covariants in $n \neq 4$ exceeds the number of independent covariants in $n=4$ where one has four independent covariants. 
The sums over $i$ in (4.12) run from 1 to 4 except for the first term (it is proportional to the Born term), where the sum runs from 1 to 6 . Below we list the color factors and analytic functions for the two 4-point functions of (4.12). For the graph (5a) we get:

$$
T_{\text {col }}=\left(T^{a} T^{b}\right)\left(T^{b} T^{a}\right),
$$

where the first parentheses in (4.13) corresponds to the summation over color indices of the massless fermion line and

$$
\begin{aligned}
& f_{1}=\ln ^{2}\left(\frac{s}{m^{2}}\right)+4 f_{l l}-4 \ln \left(\frac{s}{m^{2}}\right) \ln \left(-\frac{t}{m^{2}}\right)+2 \zeta(2), \\
& f_{2}=\ln \left(-\frac{t}{m^{2}}\right), \quad f_{3}=\ln \left(\frac{s}{m^{2}}\right), \quad f_{4}=f_{\text {lld }}, \quad f_{5}=f_{l l}, \quad f_{6}=1 .
\end{aligned}
$$

The color factor and the corresponding functions for the second box graph (5b) are

$$
T_{\text {col }}=\left(T^{a} T^{b}\right)\left(T^{a} T^{b}\right),
$$

and all the functions are obtained from the ones in (4.14) by the simple interchange $t \rightarrow u$, e.g.:

$$
\begin{aligned}
& f_{1}=\ln ^{2}\left(\frac{s}{m^{2}}\right)+4 \mathrm{f}_{11}(t \rightarrow u)-4 \ln \left(\frac{s}{m^{2}}\right) \ln \left(-\frac{u}{m^{2}}\right)+2 \zeta(2), \\
& f_{2}=\ln \left(-\frac{u}{m^{2}}\right), \quad f_{3}=\ln \left(\frac{s}{m^{2}}\right), \quad f_{4}=f_{1 l d}, \quad f_{5}=f_{11}(t \rightarrow u), \quad f_{6}=1 .
\end{aligned}
$$

The coefficients $h_{i}, h_{i}^{(j)}$ are given in Appendix B of this paper. However, there exists a partial symmetry for these box diagrams, which allows one to express most coefficients for the box graph (5b) through the ones of the box graph (5a). In particular, starting from the coefficients $h_{i}^{(j)}$ with superscript $j \geq 2$, we find the following general relations for all these coefficients:

$$
\begin{aligned}
& h_{i}^{(j)}[(5 \mathrm{~b})]=-h_{i}^{(j)}[(5 \mathrm{a})](t \rightarrow u), \quad j=2,4 ; \quad i=1 \div 4 ; \\
& h_{i}^{(j)}[(5 \mathrm{~b})]=h_{i}^{(j)}[(5 \mathrm{a})](t \rightarrow u), \quad j=3,5,6 ; \quad i=1 \div 4 .
\end{aligned}
$$

Consequently, for the graph (5b) only the coefficients $h_{i}$ and $h_{i}^{(1)}$ are presented in Appendix B. We should also mention that all the one-loop matrix elements of this chapter must be multiplied by the common factor (2.4).

At the end of this section we must again say a few words about the DRED result. In line of the discussion in the previous section one has a cancellation of differences between DREG and DRED for the heavy quark loops: the two massive self-energy diagrams (5h) and (5i) versus the heavy quark vertex graphs (5e) and (5f). Concerning the differences arising from the two massless vertex diagrams $(5 \mathrm{c}),(5 \mathrm{~d})$ and the off-shell gluon self-energy $(5 \mathrm{~g})$, these differences remain, leading to an overall difference

$$
\Delta(q \bar{q} \rightarrow Q \bar{Q})=B_{q \bar{q}} / 3 .
$$


First we stress that as the above difference is proportional to the LO Born term, it is manifestly gauge invariant. Secondly, it can be considered as a conversion term between DREG and DRED for the virtual corrections for this subprocess.

\section{ABSORPTIVE PARTS}

In the previous sections we have been writing down expressions for the real parts of the matrix elements. However, for some physical applications as e.g. the calculation of $T$ odd observables the imaginary (or absorptive) parts of the one-loop amplitudes are needed as well. In addition, the NNLO calculation of heavy quark production involves also the product of the NLO one-loop contributions including their imaginary parts.

In order to derive the imaginary parts of our matrix elements we have proceeded in two different ways. Firstly, we applied Cutkosky rules [24] to the scalar integrals that enter the Passarino-Veltman decomposition for our tensor integrals and calculated their imaginary parts directly, as discontinuities across the unitarity cut in the physical s-channel of the corresponding Feynman diagram. The result of these considerations may be cast into the form of an effective rule which states that the imaginary parts of our scalar integrals, and consequently the full matrix elements including real and imaginary pieces, can be obtained with the four simple substitutions in all of our expressions for matrix elements:

$$
\begin{aligned}
\ln \left(\frac{s}{m^{2}}\right) \rightarrow \ln \left(\frac{s}{m^{2}}\right)-i \pi, & \ln (x) \rightarrow \ln (x)+i \pi, \\
\ln ^{2}\left(\frac{s}{m^{2}}\right) \rightarrow \ln ^{2}\left(\frac{s}{m^{2}}\right)-2 i \pi \ln \left(\frac{s}{m^{2}}\right), & \ln ^{2}(x) \rightarrow \ln ^{2}(x)+2 i \pi \ln (x) .
\end{aligned}
$$

Note that the other logarithmic and dilog functions do not possess any imaginary parts. Secondly, to double-check our prescription (5.1), we have carefully traced the sign of the causal term $+i 0$ in the propagators of the scalar integrals during the whole course of their derivation. We came to the conclusion that all the analytic functions are well defined in the physical region except for the two logarithms, which have negative arguments. They enter the expressions for the scalar integrals as $\ln \left(-\frac{s+i 0}{m^{2}}\right)$ and $\ln (-x+i 0)$. In other words, in all the expressions for our amplitudes the above two logarithms would actually appear (instead of $\ln \left(s / m^{2}\right)$ and $\left.\ln (x)\right)$, if we had not already extracted $-\pi^{2}$ terms from $\ln ^{2}\left(-s / m^{2}-i 0\right)$ and $\ln ^{2}(-x+i 0)$ and considered them in the real parts of the amplitudes, and this is fully consistent with (5.1).

In case one desires to perform crossing and obtain results for other subprocesses many of the analytic functions of (3.7), (3.9), (3.11), (4.14) and (4.16) will develop imaginary parts. The rules for deriving such imaginary contributions were considered in detail in Ref. [25]. To apply those rules one needs to know the signs of the causal terms in our analytic functions. Thus, the only remaining thing to write down is a relative sign between the parameters of the analytic functions and the causal term $i 0$ for the case of our kinematics:

$$
s \rightarrow s+i 0, \quad t \rightarrow t+i 0, \quad u \rightarrow u+i 0, \quad x \rightarrow x-i 0 .
$$




\section{CONCLUSIONS}

In this paper we have presented complete analytic results for the one-loop contributions to heavy flavor production in a closed form, including their absorptive parts. These include the one-loop matrix elements of the relevant partonic subprocesses (1.1) and (1.2) that are presented here for the first time ${ }^{2}$. We have also indicated the way of deriving matrix elements for the other processes that can be obtained from those presented in this paper by crossing to different production channels. Our results are relevant not only for various NLO applications, but produce that part of the next-to-next-to-leading order corrections to heavy flavor production corresponding to the square of the one-loop matrix elements. Of course, to conclude the latter task the $\mathrm{O}\left(\varepsilon^{2}\right)$ expansion of the relevant NLO matrix element is also required. We reserve this task for a future publication.

\section{ACKNOWLEDGEMENTS}

We are grateful to A. Davydychev and A. Pivovarov for very informative discussions. Z. M. would like to thank the Particle Theory group of the Institut für Physik, Universität Mainz, and the Physics Department of McGill University for hospitality. The work of Z. M. was supported by a DFG grant under contract 436 GEO 17/3/02.

\section{APPENDIX A}

Here we present the coefficients of the box contributions for the gluon fusion subprocess appearing in eq. (3.1).

Let us introduce the notation:

$$
\begin{aligned}
z_{1} & \equiv m^{2} s-t^{2}, \quad z_{2} \equiv s+2 t, \quad z_{t} \equiv 2 m^{2}+t, \quad z_{u} \equiv 2 m^{2}+u \\
D & \equiv m^{2} s-u t .
\end{aligned}
$$

First we list coefficients for the abelian type of box diagram (2a1):

$$
\begin{aligned}
b_{1}= & (1 / \beta+\beta) / \varepsilon t, \quad b_{2}=\left(z_{u}-u \beta\right) / 2 D, \quad b_{3}=z_{t} z_{u} / t \beta D, \quad b_{4}=2 z_{u} / D, \\
b_{5}= & \left(4 u \beta-3 z_{u}\right) / D, \quad b_{6}=b_{7}=0, \quad b_{8}=-2(1 / \beta+\beta) / t ; \\
b_{11}^{(p)}= & 2 z_{t} \beta / D, \quad b_{21}^{(p)}=\left(b_{01}^{(p)}+b_{31}^{(p)}\right) / 2, \quad b_{31}^{(p)}=s t^{2} \beta^{3} / D^{2}, \\
b_{41}^{(p)}= & -2 z_{t}\left(2 m^{2} / t-s t \beta^{2} / D\right) / D, \quad b_{51}^{(p)}=-3 b_{01}^{(p)}-4 b_{31}^{(p)}, \quad b_{61}^{(p)}=2 z_{t}^{2} / T D, \quad b_{71}^{(p)}=0, \\
& \text { with } \quad b_{01}^{(p)}=\left(z_{t} D+t z_{1} \beta^{2}\right) / D^{2} ;
\end{aligned}
$$

\footnotetext{
${ }^{2}$ We would be happy to provide our one-loop results in REDUCE format. Please contact Z.M. by e-mail.
} 


$$
\begin{aligned}
& b_{12}^{(p)}=-4 m^{2} \beta\left(z_{t}+z_{1} / s\right) / D^{2}, \quad b_{22}^{(p)}=\left(b_{02}^{(p)}+b_{32}^{(p)}\right) / 2, \\
& b_{32}^{(p)}=4 m^{2} t \beta\left(D(2+t / s)-s t \beta^{2}\right) / D^{3}, \quad b_{42}^{(p)}=-8 m^{2}\left(s \beta^{2}\left(T D+t^{2} z_{t}\right)+m^{2} z_{2} T D / t\right) / t D^{3}, \\
& b_{52}^{(p)}=-3 b_{02}^{(p)}-4 b_{32}^{(p)}, \quad b_{62}^{(p)}=4 m^{2}\left(2 D / t+s \beta^{2}-4 t u / s-t^{2} / T\right) / D^{2}, \quad b_{72}^{(p)}=4 z_{t} u / s t D, \\
& \text { with } \quad b_{02}^{(p)}=4 m^{2} t\left(-z_{1} \beta^{2}+u z_{2} D / s^{2}\right) / D^{3} \text {; } \\
& b_{13}^{(p)}=-4 \beta\left(2\left(2 m^{2}-s\right) z_{t} D / s^{2} \beta^{2}-2 m^{2} s \beta^{2}-3\left(m^{2} / s+1\right) D+2 m^{2} t z_{2} / s\right) / D^{2}, \\
& b_{23}^{(p)}=\left(b_{03}^{(p)}+b_{33}^{(p)}\right) / 2, \quad b_{33}^{(p)}=-2 z_{t}\left(t\left(\beta+2 m^{2} z_{2} / s^{2} \beta\right) D+2 m^{2} u z_{2} \beta\right) / D^{3} \text {, } \\
& b_{43}^{(p)}=-4\left(2 m^{4} z_{2} s \beta^{2}+\left(4 m^{2} T D / t^{2}-z_{t}\left(2 m^{4} / t-t\right)+2 m^{2} z_{2}\right) D\right) / D^{3} \text {, } \\
& b_{53}^{(p)}=-3 b_{03}^{(p)}-4 b_{33}^{(p)}, \quad b_{63}^{(p)}=4 z_{t}\left(2\left(D / t-z_{1} / s+2 z_{2}\right)+3 t u / T-t^{2}\left(m^{2}+2 t\right) / T^{2}\right) / D^{2} \text {, } \\
& b_{73}^{(p)}=4 z_{t}\left(u / s-m^{2} / T\right) / t D, \\
& \text { with } \quad b_{03}^{(p)}=2\left(2 m^{2} u z_{1} \beta^{2}-\left(2 m^{2} T-2 m^{2} u\left(u^{2}+t^{2}\right) / s^{2}+t^{2} \beta^{2}\right) D\right) / D^{3} \text {; } \\
& b_{14}^{(p)}=-4 \beta\left(z_{t} D / s \beta^{2}+3 T^{2}-t^{2}\left(m^{2}-t\right) / s\right) / D^{2}, \quad b_{24}^{(p)}=\left(b_{04}^{(p)}+b_{34}^{(p)}\right) / 2, \\
& b_{34}^{(p)}=2 t\left(4 m^{2} D^{2} / s^{2} \beta+2 m^{2} u \beta D / s-t\left(3 s T+t^{2}\right) \beta^{3}\right) / D^{3} \text {, } \\
& b_{44}^{(p)}=4 z_{t}\left(\left(2 m^{4} / t-t\right) D-2 s t T \beta^{2}\right) / D^{3}, \\
& b_{54}^{(p)}=-3 b_{04}^{(p)}-4 b_{34}^{(p)}, \quad b_{64}^{(p)}=-8\left(z_{t} / s+t^{2} \beta^{2} / D\right) / D, \quad b_{74}^{(p)}=-4 z_{t} / s D, \\
& \text { with } \quad b_{04}^{(p)}=2\left(2 t\left(m^{2} t^{2}-s T^{2}\right) \beta^{2}-\left(6 m^{2} D / s+t z_{t}+2 m^{2} t^{2} z_{2} / s^{2}\right) D\right) / D^{3} \text {; } \\
& b_{15}^{(p)}=-4 m^{2} \beta\left(z_{t}+z_{1} / s\right) / D^{2}, \quad b_{25}^{(p)}=\left(b_{05}^{(p)}+b_{35}^{(p)}\right) / 2, \\
& b_{35}^{(p)}=4 m^{2} t \beta\left((2+t / s) D-s t \beta^{2}\right) / D^{3}, \quad b_{45}^{(p)}=-8 m^{2}\left(T\left(D-t z_{t}\right) D / t^{2}+s t z_{t} \beta^{2}\right) / D^{3}, \\
& b_{55}^{(p)}=-3 b_{05}^{(p)}-4 b_{35}^{(p)}, \quad b_{65}^{(p)}=4 m^{2}\left(2 D / t+s \beta^{2}-4 t u / s-t^{2} / T\right) / D^{2}, \quad b_{75}^{(p)}=4 z_{t} u / s t D, \\
& \text { with } \quad b_{05}^{(p)}=4 m^{2} t\left(u z_{2} D / s^{2}-z_{1} \beta^{2}\right) / D^{3} \text {; } \\
& b_{11}^{(m)}=-2\left(4 m^{2} / s-3+t z_{t} / D\right) / s \beta, \quad b_{21}^{(m)}=\left(b_{01}^{(m)}+b_{31}^{(m)}\right) / 2, \\
& b_{31}^{(m)}=t\left(4 m^{2} u / s^{2} \beta+\left(2 s T+t^{2}\right) \beta / D\right) / D, \quad b_{41}^{(m)}=-2\left(2 m^{2} T / t-z_{t}\left(2 s T+t^{2}\right) / D\right) / D, \\
& b_{51}^{(m)}=-3 b_{01}^{(m)}-4 b_{31}^{(m)}, \quad b_{61}^{(m)}=2\left(z_{t}-2 t u / s\right) / D, \quad b_{71}^{(m)}=0, \\
& \text { with } \quad b_{01}^{(m)}=\left(2\left(T-m^{2} u / s\right)-t^{2} z_{t} / D\right) / D \text {; } \\
& b_{12}^{(m)}=2\left(\beta-t z_{u} / \beta D\right) / s, \quad b_{22}^{(m)}=\left(b_{02}^{(m)}+b_{32}^{(m)}\right) / 2, \\
& b_{32}^{(m)}=-t^{2}\left(4 m^{2} / s^{2} \beta-u \beta / D\right) / D, \quad b_{42}^{(m)}=-2\left(2 m^{2}\left(m^{2} / t+2\right)-t u z_{t} / D\right) / D, \\
& b_{52}^{(m)}=-3 b_{02}^{(m)}-4 b_{32}^{(m)}, \quad b_{62}^{(m)}=2\left(2 D / s+2 s+t+t^{2} / T\right) / D, \quad b_{72}^{(m)}=0, \\
& \text { with } b_{02}^{(m)}=\left(-2 m^{2}(1-t / s)+t u z_{t} / D\right) / D \text {; } \\
& b_{11}^{(n)}=-2\left(2-2 m^{2} t z_{2} / s D+3 m^{2} s \beta^{2} / D\right) / s \beta, \quad b_{21}^{(n)}=\left(b_{01}^{(n)}+b_{31}^{(n)}\right) / 2, \\
& b_{31}^{(n)}=\left(2\left(T+2 m^{2} t^{2} / s^{2}\right) / \beta-\left(2 m^{2} z_{1}-t^{2} u\right) \beta / D\right) / D \text {, } \\
& b_{41}^{(n)}=2\left(2 m^{2}\left(m^{2} / t+2\right) D-2 m^{2} s t \beta^{2}-t^{2} z_{u}\right) / D^{2}, \quad b_{51}^{(n)}=-3 b_{01}^{(n)}-4 b_{31}^{(n)} \text {, } \\
& b_{61}^{(n)}=-2\left(6 m^{2}+2 u^{2} / s-5 m^{2} t / T\right) / D, \quad b_{71}^{(n)}=0, \\
& \text { with } \quad b_{01}^{(n)}=\left(2 m^{2}(1-t / s)-2 m^{2} s t \beta^{2} / D-t^{2} z_{u} / D\right) / D \text {; }
\end{aligned}
$$




$$
\begin{aligned}
& b_{12}^{(n)}=-2\left(2 t / s-2 t^{2} z_{t} / s D+3 s T \beta^{2} / D\right) / s \beta, \quad b_{22}^{(n)}=\left(b_{02}^{(n)}+b_{32}^{(n)}\right) / 2, \\
& b_{32}^{(n)}=\left(2\left(-2 m^{2} t u / s^{2}-T\right) / \beta-\left(2 m^{2} s T-t^{2} z_{t}\right) \beta / D\right) / D \text {, } \\
& b_{42}^{(n)}=2\left(2 m^{2} T D / t-2 s t T \beta^{2}-t^{2} z_{t}\right) / D^{2}, \quad b_{52}^{(n)}=-3 b_{02}^{(n)}-4 b_{32}^{(n)} \text {, } \\
& b_{62}^{(n)}=-2\left(3 z_{t}+2 t^{2} / s\right) / D, \quad b_{72}^{(n)}=0, \\
& \text { with } \quad b_{02}^{(n)}=t\left(2 m^{2} / s-1-z_{1} \beta^{2} / D+t z_{u} / D\right) / D \text {; } \\
& c_{1}=4 / s \beta, \quad c_{2}=\left(c_{3}+c_{4} / 2\right) / 2, \quad c_{3}=-\left(s \beta+z_{t} / \beta+2 t z_{2} / s \beta\right) / D, \\
& c_{4}=2(s+3 t) / D, \quad c_{5}=-4 c_{3}-3 c_{4} / 2, \quad c_{6}=c_{7}=0 \text {; } \\
& d_{11}=4 z_{t} / s \beta D, \quad d_{21}=\left(d_{31}+d_{41} / 2\right) / 2, \quad d_{31}=-2 T(2 / s \beta+s \beta / D) / D, \\
& d_{41}=4 T z_{2} / D^{2}, \quad d_{51}=-4 d_{31}-3 d_{41} / 2, \quad d_{61}=-4 / D, \quad d_{71}=0 ; \\
& d_{12}=4 z_{u} / s \beta D, \quad d_{22}=\left(d_{32}+d_{42} / 2\right) / 2, \quad d_{32}=2\left(2 m^{2} z_{1}-t u z_{2}\right) / s \beta D^{2}, \\
& d_{42}=4 t z_{u} / D^{2}, \quad d_{52}=-4 d_{32}-3 d_{42} / 2, \quad d_{62}=-4 m^{2} / T D, \quad d_{72}=0 ; \\
& g_{11}=2(t \beta / D-4 / s \beta), \quad g_{21}=\left(g_{31}+g_{41} / 2\right) / 2, \quad g_{31}=t\left(4 z_{t} D / s \beta-t z_{2} \beta\right) / D^{2}, \\
& g_{41}=-2\left(6 T D-s t^{2} \beta^{2}\right) / D^{2}, \quad g_{51}=-4 g_{31}-3 g_{41} / 2, \quad g_{61}=2 t z_{t} / T D, \quad g_{71}=0 ; \\
& g_{12}=4 \beta\left(1+\left(m^{2} z_{2} / s-u\right) / s \beta^{2}-2 t^{2} u / s D\right) / D, \quad g_{22}=\left(g_{32}+g_{42} / 2\right) / 2, \\
& g_{32}=2\left(-2 m^{2} t\left(6 D-t z_{2}\right) / s^{2} \beta+\left(2 m^{2} s+t^{2}\right) \beta+2 m^{2} t^{2} z_{2} \beta / D\right) / D^{2} \text {, } \\
& g_{42}=4\left(2 m^{2}\left(m^{2}-s\right)+z_{t}^{2}-2 m^{2} s t^{2} \beta^{2} / D\right) / D^{2}, \quad g_{52}=-4 g_{32}-3 g_{42} / 2, \\
& g_{62}=4\left(4 m^{2} s-t u(3+4 t / s)+3 m^{2} t^{2} / T\right) / D^{2}, \quad g_{72}=4 u / s D ; \\
& g_{13}=-4\left(D\left(m^{2}+6 m^{2} t / s+s\right) / s \beta+2 t U \beta\right) / D^{2}, \quad g_{23}=\left(g_{33}+g_{43} / 2\right) / 2, \\
& \left.g_{33}=-2\left(\left(8 m^{2}+t\right) D / s \beta+2 m^{2} t^{2} z_{2} / s^{2} \beta-t\left(3 m^{2}+t^{2} / s\right) \beta\right)+2 m^{2} t u z_{2} \beta / D\right) / D^{2} \text {, } \\
& g_{43}=4\left(6 m^{4}-t z_{t}+2 m^{2} s t u \beta^{2} / D\right) / D^{2}, \quad g_{53}=-4 g_{33}-3 g_{43} / 2 \text {, } \\
& g_{63}=4\left(4 D+2 t(2+t / s) z_{2}-3 t^{2} z_{2} / T+t^{3} z_{t} / T^{2}\right) / D^{2}, \quad g_{73}=-4\left(1-m^{2} u / D\right) / s T ; \\
& g_{14}=4\left(m^{2} D(2 t / s-1) / \beta+2 t^{3} \beta\right) / s D^{2}, \quad g_{24}=\left(g_{34}+g_{44} / 2\right) / 2 \text {, } \\
& g_{34}=2\left(2\left(2 m^{2} u / s+z_{2}\right) D / s \beta-2 m^{2} t^{2} z_{2} / s^{2} \beta+t z_{t} \beta+2 t^{3} z_{t} \beta / D\right) / D^{2} \text {, } \\
& g_{44}=4\left(2 T\left(3 m^{2}-s\right)+t^{2}-2 s t^{2} T \beta^{2} / D\right) / D^{2}, \quad g_{54}=-4 g_{34}-3 g_{44} / 2 \text {, } \\
& g_{64}=8\left(s T-2 t^{2} u / s\right) / D^{2}, \quad g_{74}=-4 t / s D ; \\
& g_{15}=4\left(D\left(m^{2} z_{2} / s-t\right) / \beta-2 t^{2} u \beta\right) / s D^{2}, \quad g_{25}=\left(g_{35}+g_{45} / 2\right) / 2, \\
& g_{35}=2\left(2 u\left(2 m^{2}-s\right) D / s^{2} \beta+t(2 T-t u / s) \beta+t^{2} z_{t} / s \beta+2 m^{2} t^{2} z_{2} \beta / D\right) / D^{2} \text {, } \\
& g_{45}=4\left(6 m^{4}-2 m^{2} s-t^{2}+2 s t T u \beta^{2} / D\right) / D^{2}, \quad g_{55}=-4 g_{35}-3 g_{45} / 2 \text {, } \\
& g_{65}=4\left(2 D-t(1+4 t / s) u+m^{2} t^{2} / T\right) / D^{2}, \quad g_{75}=4 u / s D \text {. }
\end{aligned}
$$

Now we list coefficients for the nonabelian box diagram (2a2):

$$
\begin{aligned}
b_{1} & =-2 / \varepsilon t, \quad b_{2}=0, \quad b_{3}=(s / 2-t) / D, \quad b_{4}=-\left(z_{2} / 2+z_{t}\right) / \beta D, \\
b_{5} & =-10 / t, \quad b_{6}=-2 z_{t} / t T, \quad b_{7}=2\left(1 / \varepsilon^{2}+1 / \varepsilon+2\right) / t, \quad b_{8}=-4 / t, \quad b_{9}=4 / t ; \\
b_{11}^{(p)} & =-1 / \varepsilon s-2\left(m^{2}+t^{2} / s\right) / D, \quad b_{21}^{(p)}=1 / 2 s, \quad b_{31}^{(p)}=t^{2}\left(z_{2} / 2 D-2 / s\right) / D, \\
b_{41}^{(p)} & =\left(m^{2}(s-4 t) D / s+s t z_{t} / 2-t^{3} z_{2} / s\right) / \beta D^{2}, \quad b_{51}^{(p)}=-4 / s,
\end{aligned}
$$




$$
\begin{aligned}
& b_{61}^{(p)}=-8 / \varepsilon s-2 t(2-t / T) / D, \quad b_{71}^{(p)}=\left(5 / \varepsilon^{2}+2 / \varepsilon+4\right) / s, \quad b_{81}^{(p)}=8 / s ; \\
& b_{12}^{(p)}=-4 m^{2}\left(2 t / D-1 / s \beta^{2}\right) / D, \quad b_{22}^{(p)}=0, \quad b_{32}^{(p)}=2 z_{1} m^{2} t / D^{3}, \\
& b_{42}^{(p)}=-2 m^{2}\left(2 m^{2} / s^{2} \beta^{2}+t\left(m^{2}(s+4 t)+t^{2}\right) / D^{2}\right) / \beta D, \quad b_{52}^{(p)}=0, \\
& b_{62}^{(p)}=4\left(m^{2}(s+3 t)+m^{4} t / T\right) / D^{2}, \quad b_{72}^{(p)}=-4 u / s D, \quad b_{82}^{(p)}=0 ; \\
& b_{13}^{(p)}=4\left(2 m^{2} u / D+\left(m^{2}+(3+4 t / s)\left(2 m^{2}-s\right)\right) / s \beta^{2}\right) / D, \quad b_{23}^{(p)}=0, \\
& b_{33}^{(p)}=-z_{t}\left(2 m^{2} s u / D-t\right) / D^{2}, \\
& b_{43}^{(p)}=\left(4 m^{2}\left(m^{2}+z_{2}\right) / s^{2} \beta^{2}-2 m^{2} / s-2 m^{2} s / D+t^{2} / D-2 m^{4} s^{2} \beta^{2} / D^{2}\right) / \beta D, \\
& b_{53}^{(p)}=0, \quad b_{63}^{(p)}=4\left(D t^{2} / T^{2}+2 t^{2}(s-t) / T+10 m^{2} s+4 t z_{t}-4 t^{2} u / s\right) / D^{2}, \\
& b_{73}^{(p)}=4(2+t / s-t / T) / D, \quad b_{83}^{(p)}=0 ; \\
& b_{14}^{(p)}=4\left(\left(3 m^{2}+2 t\right) / \beta^{2}+2 t^{3} / D\right) / s D, \quad b_{24}^{(p)}=0, \quad b_{34}^{(p)}=-t\left(2 m^{2}+3 t+2 t^{2} z_{t} / D\right) / D^{2}, \\
& b_{44}^{(p)}=-\left(3 m^{2}+t+\left(3 m^{2}+2 t\right) / \beta^{2}+t\left(m^{2} s-3 t^{2}\right) / D+2 s t^{4} \beta^{2} / D^{2}\right) / s \beta D, \\
& b_{54}^{(p)}=0, \quad b_{64}^{(p)}=-8 t^{2} z_{2} / s D^{2}, \quad b_{74}^{(p)}=4 t / s D, \quad b_{84}^{(p)}=0 ; \\
& b_{15}^{(p)}=-4 m^{2}\left(2 t / D-1 / s \beta^{2}\right) / D, \quad b_{25}^{(p)}=0, \quad b_{35}^{(p)}=2 m^{2} t z_{1} / D^{3}, \\
& b_{45}^{(p)}=-2 m^{2}\left(2 m^{2} / s^{2} \beta^{2}+t\left(m^{2} z_{2}+t z_{t}\right) / D^{2}\right) / \beta D, \quad b_{55}^{(p)}=0, \\
& b_{65}^{(p)}=4\left(m^{2} s+4 m^{2} t-t^{2}+t^{3} / T\right) / D^{2}, \quad b_{75}^{(p)}=-4 u / s D, \quad b_{85}^{(p)}=0 ; \\
& b_{11}^{(m)}=2 / \varepsilon s+\left(T-t^{2} / s-4 m^{2} z_{t} / s \beta^{2}\right) / D, \quad b_{21}^{(m)}=-1 / s, \\
& b_{31}^{(m)}=t\left(z_{2} / s+t\left(2 m^{2}+t / 2\right) / D\right) / D \text {, } \\
& b_{41}^{(m)}=\left(2 m^{2} D / s \beta^{2}-m^{2} t z_{2} / s \beta^{2}+2 m^{2} z_{2}+t\left(2 m^{2}-u\right)+3 s t^{3} \beta^{2} / 2 D+t^{3} z_{t} / D\right) / s \beta D, \\
& b_{51}^{(m)}=8 / s, \quad b_{61}^{(m)}=8 / \varepsilon s+2 t(3+4 t / s) / D, \quad b_{71}^{(m)}=-3\left(2 / \varepsilon^{2}+1 / \varepsilon+2\right) / s, \quad b_{81}^{(m)}=-8 / s ; \\
& b_{12}^{(m)}=2 / \varepsilon s+\left(3\left(m^{2}+t u / s\right)-4 m^{2} t z_{2} / s^{2} \beta^{2}\right) / D, \quad b_{22}^{(m)}=-1 / s, \\
& b_{32}^{(m)}=t(1+4 t / s+t u(3 / 2+2 t / s) / D) / D \text {, } \\
& b_{42}^{(m)}=-\left(T-t z_{t} / s-m^{2} t z_{2} / s^{2} \beta^{2}+t^{2} u z_{2} / 2 s D+m^{2} s t \beta^{2} / D\right) / \beta D, \\
& b_{52}^{(m)}=8 / s, \quad b_{62}^{(m)}=2\left((1 / t+4 / s) / \varepsilon-2\left(z_{1} / t-t z_{2} / s\right) / D+m^{2} t / T D\right), \\
& b_{72}^{(m)}=-\left(1 / \varepsilon^{2} t+6 / \varepsilon^{2} s-2 / \varepsilon t+3 / \varepsilon s-2(2 / t-3 / s)\right), \quad b_{82}^{(m)}=-2(1 / t+4 / s) ; \\
& b_{11}^{(n)}=-2 / \varepsilon s+\left(5 m^{2}-2 t+t^{2} / s+4 z_{t} / \beta^{2}+t z_{2} / s \beta^{2}\right) / D, \quad b_{21}^{(n)}=1 / s, \\
& b_{31}^{(n)}=-\left(2 m^{2}-3 t\right) / D+t^{2} u(1 / 2-2 u / s) / D^{2} \text {, } \\
& b_{41}^{(n)}=-\left(4 z_{t}\left(s z_{t}-3 m^{2} t\right) / s^{2} \beta^{2}-6 m^{4} z_{2} / D-m^{2} s t \beta^{2} / D-t\left(2 m^{2} / s+1\right)\right) / 2 \beta D, \\
& b_{51}^{(n)}=-b_{52}^{(m)}, \quad b_{61}^{(n)}=-b_{62}^{(m)}-16 m^{4} / T D, \quad b_{71}^{(n)}=-b_{72}^{(m)}, \quad b_{81}^{(n)}=-b_{82}^{(m)} ; \\
& b_{12}^{(n)}=-2 / \varepsilon s-\left(\left(8 m^{2}+s\right) z_{t} / s \beta^{2}-m^{2}-t^{2} / s\right) / D, \quad b_{22}^{(n)}=1 / s, \\
& b_{32}^{(n)}=-\left(2 / s-s t T / D^{2}-t^{2}\left(2 m^{2}+t / 2\right) / D^{2}\right) \text {, } \\
& b_{42}^{(n)}=\left(3 m^{2} z_{t} / s \beta^{2}-t\left(2 T / s+1-\left(2 m^{2}-t / 2\right) z_{t} / D\right)\right) / \beta D \text {, } \\
& b_{52}^{(n)}=-b_{51}^{(m)}, \quad b_{62}^{(n)}=-b_{61}^{(m)}-16 m^{2} / D, \quad b_{72}^{(n)}=-b_{71}^{(m)}, \quad b_{82}^{(n)}=-b_{81}^{(m)} \text {; }
\end{aligned}
$$




$$
\begin{aligned}
& c_{1}=-2 / s \beta^{2}, \quad c_{2}=0, \quad c_{3}=-s / 2 D, \quad c_{4}=1 / 2 s \beta^{3}+z_{2} / 2 \beta D, \quad c_{5}=0, \\
& c_{6}=-2 / T, \quad c_{7}=c_{8}=0 \text {; } \\
& d_{11}=-8 z_{t} / s \beta^{2} D, \quad d_{21}=0, \quad d_{31}=\left(-s T+t^{2}\right) / D^{2}, \\
& d_{41}=\left(z_{2} / s \beta^{2}+2 t z_{t} / D\right) / \beta D, \quad d_{51}=0, \quad d_{61}=-8 / D, \quad d_{71}=d_{81}=0 ; \\
& d_{12}=-8 z_{u} / s \beta^{2} D, \quad d_{22}=0, \quad d_{32}=-\left(m^{2} s+t u\right) / D^{2}, \\
& d_{42}=z_{2}\left(-1 / s \beta^{2}+2 m^{2} / D\right) / \beta D, \quad d_{52}=0, \quad d_{62}=-8 m^{2} / T D, \quad d_{72}=d_{82}=0 \\
& g_{11}=2 z_{t} / \beta^{2} D, \quad g_{21}=0, \quad g_{31}=-s t^{2} / 2 D^{2} \text {, } \\
& g_{41}=-\left(2 / s+m^{2} z_{2} / s \beta^{2} D+s t z_{t} / 2 D^{2}\right) / \beta, \quad g_{51}=0, \quad g_{61}=-2 t^{2} / T D, \quad g_{71}=g_{81}=0 ; \\
& g_{12}=-4\left(2+t / s-2 U / s \beta^{2}-3 m^{2} z_{2} / s^{2} \beta^{4}-2 m^{2} t z_{2} / s \beta^{2} D\right) / D, \quad g_{22}=0, \\
& g_{32}=s\left(4 m^{2}+t z_{t} / s+2 t^{3} u / s D\right) / D^{2} \text {, } \\
& g_{42}=\left(2\left(2 m^{4}+2 m^{2} u-t T\right) / D+2\left(4 m^{4} / s+m^{2}+2 m^{2} t / s-t\right) / s \beta^{2}+t^{2} z_{2} / s \beta^{2} D\right. \\
& \left.-12 m^{4} z_{2} / s^{3} \beta^{4}-2 t^{3} z_{u} / D^{2}\right) / \beta D, \\
& g_{52}=0, \quad g_{62}=4\left(2 D(3+t / s)+3 t u+5 m^{2} t^{2} / T\right) / D^{2}, \quad g_{72}=4 z_{u} / s \beta^{2} D, \quad g_{82}=0 ; \\
& g_{13}=-4\left(2+t / s+\left(2 m^{2}-u\right) / s \beta^{2}+3 m^{2} z_{2} / s^{2} \beta^{4}-2 t U z_{2} / s \beta^{2} D\right) / D, \quad g_{23}=0, \\
& g_{33}=s\left(4 m^{2}+t z_{t} / s-2 m^{2} t u / D\right) / D^{2} \text {, } \\
& g_{43}=\left(2\left(2 m^{4}-s z_{t}-2 t^{2}+t^{3} / s\right) / D+2\left(4 m^{4} / s+3 m^{2}+16 m^{2} t / s\right) / s \beta^{2}-t^{2} z_{2} / s \beta^{2} D\right. \\
& \left.+12 m^{4} z_{2} / s^{3} \beta^{4}-2 t^{2}\left(t z_{u}+z_{2} u\right) / D^{2}\right) / \beta D, \\
& g_{53}=0, \quad g_{63}=4\left(2(2+t / s)+2 t^{2} U / T D-t^{2} / T^{2}\right) / D, \quad g_{73}=4\left(t / T+z_{t} / s \beta^{2}\right) / D, \\
& g_{83}=0 \\
& g_{14}=4\left(2 m^{2}(s-2 u) / s^{2} \beta^{2}-3 m^{2} z_{2} / s^{2} \beta^{4}+2 t^{2} z_{t} / s \beta^{2} D\right) / D, \quad g_{24}=0, \\
& g_{34}=-\left(2 T u+t^{2}+2 t^{4} / D\right) / D^{2} \text {, } \\
& g_{44}=\left(3 t^{2} / D+\left(3 m^{2}+18 m^{2} t / s-2 s-8 t\right) / s \beta^{2}-8 m^{4} z_{1} / s^{2} \beta^{2} D+3 m^{2} z_{2} / s^{2} \beta^{4}\right. \\
& \left.+2 t^{4} z_{2} / s D^{2}\right) / \beta D \\
& g_{54}=0, \quad g_{64}=8\left(2+t / s+t^{2} / D\right) / D, \quad g_{74}=4 z_{t} / s \beta^{2} D, \quad g_{84}=0 ; \\
& g_{15}=4\left(2 m^{2} z_{2} / s+3 T / \beta^{2}-6 m^{2} t / s \beta^{2}+2 t^{2} z_{u} / D\right) / s \beta^{2} D, \quad g_{25}=0, \\
& g_{35}=2 / D+t z_{t} / D^{2}+2 t^{3} u / D^{3} \text {, } \\
& g_{45}=\left(2\left(2 m^{4}+t^{2} u / s-m^{2} t^{2} z_{2} / D\right) / D+t^{2} z_{2} / s \beta^{2} D-2\left(2 m^{2}\left(m^{2}-s\right)+\left(m^{2}+s\right) z_{2}\right.\right. \\
& \left.\left.+12 m^{4} z_{t} / s \beta^{2}\right) / s^{2} \beta^{2}\right) / \beta D, \\
& g_{55}=0, \quad g_{65}=4\left(-2 D u / s+t z_{2}-t^{3} / T\right) / D^{2}, \quad g_{75}=4 z_{u} / s \beta^{2} D, \quad g_{85}=0 ;
\end{aligned}
$$

Finally, the coefficients for the crossed box (2a4) are:

$$
\begin{aligned}
b_{1} & =2(2-u / t) / s, \quad b_{2}=b_{1}(t \leftrightarrow u), \quad b_{3}=\left(2 m^{2}-3 t u / s+s\right) / D, \quad b_{4}=0, \\
b_{5} & =8(3 / s-s / t u), \quad b_{6}=2 / \varepsilon u+z_{t} / t T, \quad b_{7}=b_{6}(t \leftrightarrow u), \quad b_{8}=-b_{5} / 2, \\
b_{9} & =\left(1 / \varepsilon^{2}+1 / \varepsilon+2\right) s / t u \\
b_{11}^{(p)} & =-2\left(z_{u} / u+2\right) / s, \quad b_{21}^{(p)}=-2\left(z_{u}-2 t\right) / s u, \quad b_{31}^{(p)}=-2 z_{u} / s u+2 t / D-t u^{2} z_{t} / s D^{2},
\end{aligned}
$$




$$
\begin{aligned}
& b_{41}^{(p)}=-4 m^{2} / t u, \quad b_{51}^{(p)}=4 b_{21}^{(p)}, \quad b_{61}^{(p)}=-4 / \varepsilon u-2 m^{2} z_{t} / T D, \\
& b_{71}^{(p)}=2 z_{u}\left(t / u+2 m^{2} / U\right) / D, \quad b_{81}^{(p)}=-2 b_{21}^{(p)}, \quad b_{91}^{(p)}=2\left(1 / \varepsilon^{2}+1 / \varepsilon+2\right) / u ; \\
& b_{12}^{(p)}=8 m^{2} / s^{2} u, \quad b_{22}^{(p)}=b_{12}^{(p)}, \quad b_{32}^{(p)}=4 m^{2}\left(2 m^{2} s(D / u-t) D+t u^{2} z_{1}\right) / s^{2} D^{3}, \\
& b_{42}^{(p)}=-8 m^{2} / t^{2} u, \quad b_{52}^{(p)}=4 b_{12}^{(p)}, \quad b_{62}^{(p)}=-4 m^{2}\left(2(1 / t-1 / s) D-u(3 t-u) / s+m^{2} t / T\right) / D^{2}, \\
& b_{72}^{(p)}=-4 m^{2}\left(m^{4} / U+m^{2}-t^{2} / s+t u / s\right) / D^{2}, \quad b_{82}^{(p)}=-2 b_{12}^{(p)}, \quad b_{92}^{(p)}=4 z_{t} / t D ; \\
& b_{13}^{(p)}=4\left(2 m^{2} / u-3\right) / s^{2}, \quad b_{23}^{(p)}=b_{13}^{(p)}, \\
& b_{33}^{(p)}=b_{13}^{(p)}-2\left(2 t u / D+\left(4 m^{2} u^{3}-t u^{2} z_{t}\right) / D^{2}-2 m^{2} t u^{3}(t-u) / D^{3}\right) / s^{2}, \\
& b_{43}^{(p)}=-8 m^{2} / t^{2} u, \quad b_{53}^{(p)}=4 b_{13}^{(p)}, \\
& b_{63}^{(p)}=-4\left(2 m^{2}((1 / t-1 / s) D+u(t+5 u) / s)+t u\left(5 m^{4}+3 t u\right) / s T+m^{2} t^{2}\left(m^{4}+t u\right) / s T^{2}\right) / D^{2}, \\
& b_{73}^{(p)}=-4\left(2 m^{2}+5 u+2 t u z_{u} / D\right) / s D, \quad b_{83}^{(p)}=-2 b_{13}^{(p)}, \quad b_{93}^{(p)}=4 m^{2} z_{t} / t T D ; \\
& b_{14}^{(p)}=4\left(2 m^{2} t / u^{2}+4 m^{2} / u+3\right) / s^{2}, \quad b_{24}^{(p)}=b_{14}^{(p)}, \\
& b_{34}^{(p)}=b_{14}^{(p)}+2\left(t\left(4 m^{2} t(t-u)-u^{2}\left(2 m^{2}+3 t\right)\right) D+2 m^{2} t^{3} u(t-u)\right) / s^{2} D^{3}, \\
& b_{44}^{(p)}=-8 m^{2} / t u^{2}, \quad b_{54}^{(p)}=4 b_{14}^{(p)}, \quad b_{64}^{(p)}=4\left(2 m^{2}+5 t+2 t u z_{t} / D\right) / s D, \\
& b_{74}^{(p)}=4\left(2 m^{2}((s / u-1) D+t(u+5 t))+u t\left(5 m^{4}+3 t u\right) / U+m^{2} u^{2}\left(m^{4}+t u\right) / U^{2}\right) / s D^{2}, \\
& b_{84}^{(p)}=-2 b_{14}^{(p)}, \quad b_{94}^{(p)}=-4 m^{2} z_{u} / u U D ; \\
& b_{15}^{(p)}=8 m^{2}(t+2 u) / s^{2} u^{2}, \quad b_{25}^{(p)}=b_{15}^{(p)}, \\
& b_{35}^{(p)}=b_{15}^{(p)}-4 m^{2}\left(t u(t-2 u) D+t^{2} u^{2}(t-u)\right) / s^{2} D^{3}, \quad b_{45}^{(p)}=-8 m^{2} / t u^{2}, \\
& b_{55}^{(p)}=4 b_{15}^{(p)}, \quad b_{65}^{(p)}=4 m^{2}\left(2 m^{2} s-u^{2}+t^{2} U / T+2 m^{2} t u / T\right) / s D^{2}, \\
& b_{75}^{(p)}=4 m^{2}\left(-2 m^{2} s(t / u+2)+3 t^{2}-u^{2}\left(m^{2}-t\right) / U\right) / s D^{2}, \quad b_{85}^{(p)}=-2 b_{15}^{(p)}, \quad b_{95}^{(p)}=-4 z_{u} / u D ; \\
& b_{11}^{(m)}=-2\left(2 m^{2}+3 t u / s\right) / s u, \quad b_{21}^{(m)}=b_{11}^{(m)}+2 / u \\
& b_{31}^{(m)}=\left(-2\left(2 m^{2} s / u+t\right)+2 s\left(2 m^{2} u-s t\right) / D-t^{3} u^{2} / D^{2}\right) / s^{2}, \quad b_{41}^{(m)}=-4 m^{2} / t u, \\
& b_{51}^{(m)}=4 b_{11}^{(m)}+16 / u, \quad b_{61}^{(m)}=4 / \varepsilon u-2(2 D-t u) / s D, \\
& b_{71}^{(m)}=2 / \varepsilon u+2\left(2 D+t^{2}\right) / s D, \quad b_{81}^{(m)}=-b_{51}^{(m)} / 2, \quad b_{91}^{(m)}=-3 / \varepsilon^{2} u ; \\
& b_{12}^{(m)}=2\left(-2 m^{2} s / u-5 t-u+u^{2} / t\right) / s^{2}, \quad b_{22}^{(m)}=2\left(-2 m^{2} / s-1+3 t^{2} / s^{2}\right) / u, \\
& b_{32}^{(m)}=-\left(2 s\left(2 m^{2} / u-1\right)+2 t\left(3 m^{2} s+t^{2}\right) / D-t^{2} u^{3} / D^{2}\right) / s^{2}, \quad b_{42}^{(m)}=b_{41}^{(m)}, \\
& b_{52}^{(m)}=4 b_{22}^{(m)}, \quad b_{62}^{(m)}=2(2 / u-1 / t) / \varepsilon-2\left(2 m^{2}(2+u / t)+t(t-2 u) / s+t^{2} / T\right) / D, \\
& b_{72}^{(m)}=2\left(t\left(-2 m^{2} s / u+t-2 u\right) / s+m^{2} u / U\right) / D, \quad b_{82}^{(m)}=-2 b_{22}^{(m)}, \\
& b_{92}^{(m)}=(1 / t-2 / u) / \varepsilon^{2}+2 s / \varepsilon t u+4 s / t u ; \\
& b_{11}^{(n)}=6(2 t+u) / s^{2}, \quad b_{21}^{(n)}=-2\left(3 t^{2} / s^{2}-1\right) / u, \\
& b_{31}^{(n)}=\left(2(2 t+3 u)+2 t\left(t^{2}+t u+5 u^{2}\right) / D+5 t^{2} u^{3} / D^{2}\right) / s^{2}, \quad b_{41}^{(n)}=0, \\
& b_{51}^{(n)}=4 b_{21}^{(n)}, \quad b_{61}^{(n)}=-4 / \varepsilon u+2\left(D / s+2 u^{2} / s+7 m^{4} / T\right) / D, \\
& b_{71}^{(n)}=2\left(t(2-t / u) z_{u}+6 m^{2} u-m^{2} s u / U\right) / s D, \quad b_{81}^{(n)}=-2 b_{21}^{(n)}, \quad b_{91}^{(n)}=2\left(1 / \varepsilon^{2}+1 / \varepsilon+2\right) / u ;
\end{aligned}
$$




$$
\begin{aligned}
& b_{12}^{(n)}=6 t / s^{2}, \quad b_{22}^{(n)}=-2\left(2 / u-3 t / s^{2}\right), \quad b_{32}^{(n)}=t\left(2\left(t^{2}+u^{2}\right) / D-2-t u\left(6 m^{2} s-t u\right) / D^{2}\right) / s^{2}, \\
& b_{42}^{(n)}=0, \quad b_{52}^{(n)}=4 b_{22}^{(n)}, \quad b_{62}^{(n)}=-4 / \varepsilon u+2\left(8 m^{2}-3 t u / s\right) / D \\
& b_{72}^{(n)}=-2\left(t\left(2 m^{2} t / u-4 m^{2}+3 t\right)-6 m^{2} u\left(m^{2}-t\right) / U\right) / s D, \quad b_{82}^{(n)}=-2 b_{22}^{(n)}, \quad b_{92}^{(n)}=b_{91}^{(n)} \text {; } \\
& c_{1}=c_{2}=0, \quad c_{3}=(u-t) / D, \quad c_{4}=c_{5}=0, \quad c_{6}=1 / T, \quad c_{7}=-1 / U, \quad c_{8}=c_{9}=0 ; \\
& d_{11}=d_{21}=0, \quad d_{31}=2 T u / D^{2}, \quad d_{41}=d_{51}=0, \quad d_{61}=4 / D, \quad d_{71}=-4 m^{2} / U D, \\
& d_{81}=d_{91}=0 \\
& d_{12}=d_{22}=0, \quad d_{32}=-2 t U / D^{2}, \quad d_{42}=d_{52}=0, \quad d_{62}=4 m^{2} / T D, \quad d_{72}=-4 / D, \\
& d_{82}=d_{92}=0 \\
& g_{11}=6 / s, \quad g_{21}=g_{11}, \quad g_{31}=\left(6-2 t^{2} / D-t^{2} u^{2} / D^{2}\right) / s, \quad g_{41}=0, \quad g_{51}=4 g_{11}, \\
& g_{61}=-2 m^{2} t / T D, \quad g_{71}=-2\left(t+2 m^{2} u / U\right) / D, \quad g_{81}=-2 g_{11}, \quad g_{91}=0 ; \\
& g_{12}=-12 / s^{2}, \quad g_{22}=g_{12}, \quad g_{32}=-2\left(6-u^{2}\left(4 m^{2} s+5 t^{2}\right) / D^{2}-2 t^{3} u^{3} / D^{3}\right) / s^{2}, \\
& g_{42}=0, \quad g_{52}=4 g_{12}, \quad g_{62}=4\left(5 m^{4} t / T+3 u\left(2 m^{2}-t u / s\right)\right) / D^{2}, \\
& g_{72}=-4\left(m^{2}(2 t+3 u)-t u(t+4 u) / s+m^{2} u^{2} / U\right) / D^{2}, \quad g_{82}=-2 g_{12}, \quad g_{92}=4 / D ; \\
& g_{13}=g_{12}, \quad g_{23}=g_{12}, \quad g_{33}=g_{32}+4 m^{4} s u / D^{3}, \quad g_{43}=0, \quad g_{53}=g_{52}, \\
& g_{63}=4\left(u(4 D+t u) / s+3 m^{2} t U / T+m^{4} t^{2} / T^{2}\right) / D^{2}, \quad g_{73}=4 u(2 t u-D) / s D^{2}, \\
& g_{83}=-2 g_{12}, \quad g_{93}=4 m^{2} / T D ; \\
& g_{14}=g_{12}, \quad g_{24}=g_{12}, \quad g_{34}=g_{33}+4 t^{2} u z_{u} / D^{3}, \quad g_{44}=0, \quad g_{54}=g_{52}, \\
& g_{64}=4\left(3 t+4 u+2 t^{2} u / D\right) / s D, \quad g_{74}=4 u\left(-3 t^{2} / s+3 m^{2} t / U-m^{6} / U^{2}\right) / D^{2}, \\
& g_{84}=-2 g_{12}, \quad g_{94}=g_{93}(t \leftrightarrow u) \\
& g_{15}=g_{12}, \quad g_{25}=g_{12}, \quad g_{35}=g_{34}-4 m^{4} s t / D^{3}, \quad g_{45}=0, \quad g_{55}=g_{52}, \\
& g_{65}=4\left(m^{4} t / T+u\left(2 m^{2}-3 t u / s\right)\right) / D^{2}, \quad g_{75}=g_{65}(t \leftrightarrow u), \quad g_{85}=-2 g_{12}, \quad g_{95}=g_{92} ;
\end{aligned}
$$

\section{APPENDIX B}

This Appendix contains the coefficients for the one-loop corrections to the subprocess $q \bar{q} \rightarrow Q \bar{Q}$. For the coefficients $h$ in (4.12) for the box diagram (5a) we have:

$$
\begin{aligned}
h_{1} & =\left(t^{3} / D+m^{2}+2 t^{2} / s\right) / 2 D, \quad h_{2}=2(2 / \varepsilon s+2 / s-t / D), \quad h_{3}=-2\left(1+t z_{t} / \beta^{2} D\right) / s, \\
h_{4} & =-\left(\left(m^{2} s T+t^{4} / s\right) / D-t\left(2 T+z_{t} / \beta^{2}\right) / s\right) / 2 \beta D, \quad h_{5}=-4 / s, \quad h_{6}=-2 / \varepsilon^{2} s ; \\
h_{1}^{(1)} & =2 t T / D^{2}, \quad h_{2}^{(1)}=8 t / s D, \quad h_{3}^{(1)}=-8\left(1-t z_{t} / D\right) / s^{2} \beta^{2}, \\
h_{4}^{(1)} & =2 z_{t}\left(T / D+2 m^{2} / s^{2} \beta^{2}\right) / \beta D ; \\
h_{1}^{(2)} & =-z_{1} / 4 D^{2}, \quad h_{2}^{(2)}=-z_{t} / T D, \quad h_{3}^{(2)}=1 / D, \quad h_{4}^{(2)}=\left(1-s t \beta^{2} / D\right) / 4 \beta D ; \\
h_{1}^{(3)} & =t / 8 D, \quad h_{2}^{(3)}=0, \quad h_{3}^{(3)}=0, \quad h_{4}^{(3)}=z_{t} / 8 \beta D ; \quad h_{3}^{(4)}=-4 z_{t} / s \beta^{2} D, \quad h_{4}^{(4)}=\left(t z_{1} / D+z_{t} / \beta^{2}\right) / s \beta D ; \\
h_{1}^{(4)} & =t^{2} / D^{2}, \quad h_{2}^{(4)}=-4 / D, \quad h_{3}^{(4)},
\end{aligned}
$$


$h_{1}^{(5)}=s t / 2 D^{2}, \quad h_{2}^{(5)}=2 t / T D, \quad h_{3}^{(5)}=2 z_{2} / s \beta^{2} D, \quad h_{4}^{(5)}=-\left(2 z_{t} / s \beta^{2}+t z_{2} / D\right) / 2 \beta D ;$

$h_{1}^{(6)}=h_{1}^{(5)} / 2, \quad h_{2}^{(6)}=h_{2}^{(5)} / 2, \quad h_{3}^{(6)}=h_{3}^{(5)} / 2, \quad h_{4}^{(6)}=h_{4}^{(5)} / 2$;

The nontrivial coefficients for the second box diagram (5b) are:

$$
\begin{aligned}
h_{1} & =\left(m^{2} s t / D-m^{2}-4 u-2 u^{2} / s\right) / 2 D, \quad h_{2}=-2\left(2(1 / \varepsilon+1) U / s+1-m^{2} t / D\right) / U, \\
h_{3} & =2\left(1+z_{t} t / \beta^{2} D\right) / s, \\
h_{4} & =\left(s \beta^{2}-2 z_{u}-2 D / s \beta^{2}+4 m^{2} u z_{u} / s^{2} \beta^{2}+\left(m^{2} z_{1 u}+s u t \beta^{2}\right) / D\right) / 2 \beta D, \\
h_{5} & =4 / s, \quad h_{6}=2 / \varepsilon^{2} s ; \\
h_{1}^{(1)} & =\left(m^{2} s+u z_{u}\right) / D^{2}, \quad h_{2}^{(1)}=4\left(z_{u} / U+2 u / s\right) / D, \quad h_{3}^{(1)}=-2\left(1 / \beta^{2}+1\right)(s+2 u) / s D, \\
h_{4}^{(1)} & =\left(\left(-m^{2} s \beta^{2}+u z_{1 u} / s\right) / D+\left(8 m^{4} / s+u\right) / s \beta^{2}\right) / \beta D,
\end{aligned}
$$

where for convenience we have introduced the notation:

$$
z_{1 u} \equiv m^{2} s-u^{2}=z_{1}(t \rightarrow u)
$$

The remaining coefficients for this graph can be easily obtained from the corresponding ones for the graph (5a) through the relations (4.17). 


\section{REFERENCES}

[1] G. Baum et al, COMPASS Collaboration: CERN/SPLC 96-14 and 96-30.

[2] R. Arnold et al, SLAC-PROPOSAL-E156, 1997.

[3] A. de Roeck and T. Gehrmann, DESY-Proceedings-1998-1.

[4] P. Nason, S. Dawson and R. K. Ellis, Nucl. Phys. B303, 607 (1988); Nucl. Phys. B327, 49 (1989); Erratum: ibid B335, 260 (1990).

[5] W. Beenakker, H. Kuijf, W. L. van Neerven and J. Smith, Phys. Rev. D40, 54 (1989); W. Beenakker, W. L. van Neerven, R. Meng, G. A. Schuler and J. Smith, Nucl. Phys. B351, 507 (1991).

[6] R. K. Ellis and P. Nason, Nucl. Phys. B312, 551 (1989).

[7] J. Smith and W.L. van Neerven, Nucl. Phys. B374, 36 (1992).

[8] I. Bojak and M. Stratmann, hep-ph/0112276.

[9] I. Bojak and M. Stratmanm, Phys. Lett. B433, 411 (1998).

[10] I. Bojak and M. Stratmanm, Nucl. Phys. B540, 345 (1999); Erratum: ibid B569, $694(2000)$.

[11] A.P. Contogouris, Z. Merebashvili and G. Grispos, Phys. Lett. B482, 93 (2000).

[12] Z. Merebashvili, A.P. Contogouris and G. Grispos, Phys. Rev. D62, 114509, (2000).

[13] J. H. Kühn, E. Mirkes and J. Steegborn, Z. Phys. C 57, 615, (1993).

[14] M. Drees, M. Krämer, J. Zunft and P. M. Zerwas, Phys. Lett. B306, 371 (1993).

[15] B. Kamal, Z. Merebashvili and A.P. Contogouris, Phys. Rev. D51, 4808 (1995); Erratum: ibid D55, 3229 (1997).

[16] G. Jikia and A. Tkabladze, Phys. Rev. D54, 2030 (1996).

[17] G.'t Hooft and M. Veltman, Nucl. Phys. B44, 189 (1972).

[18] W. Siegel, Phys. Lett. 84B, 193 (1979).

[19] W.C. Kuo, D. Slaven and B.L. Young, Phys. Rev. D37, 233 (1988).

[20] T. Muta, "Foundations of Quantum Chromodynamics" (World Scientific, 1987).

[21] A. I. Davydychev, P. Osland and O. V. Tarasov, Phys. Rev. D54, 4087 (1996); Erratum: ibid D59, 109901 (1999).

[22] G. Passarino and M. Veltman, Nucl. Phys. B160, 151 (1979).

[23] A. Hearn, REDUCE User's Manual Version 3.6 (Rand Corporation, Santa Monica, CA, 1995).

[24] R. E. Cutkosky, J. Math. Phys. 1, 429 (1960).

[25] J. G. Körner, B. Melić and Z. Merebashvili, Phys. Rev. D62, 096011 (2000). 


\section{FIGURE CAPTIONS}

Fig. 1. The t-, u- and s-shannel leading order (Born) graphs contributing to the gluon (curly lines) fusion amplitude. The thick solid lines correspond to the heavy quarks.

Fig. 2. The t-channel one-loop graphs contributing to the gluon fusion amplitude. Loops with dotted lines represent gluon, ghost and light and heavy quarks.

Fig. 3. The s-channel one-loop graphs contributing to the gluon fusion amplitude. Loops with dotted lines represent gluon, ghost and light and heavy quarks.

Fig. 4. The lowest order Feynman diagram contributing to the subprocess $q \bar{q} \rightarrow Q \bar{Q}$. The thick lines correspond to the heavy quarks.

Fig. 5. The one-loop Feynman diagrams contributing to the subprocess $q \bar{q} \rightarrow Q \bar{Q}$. The loop with dotted line represents gluon, ghost and light and heavy quarks. 

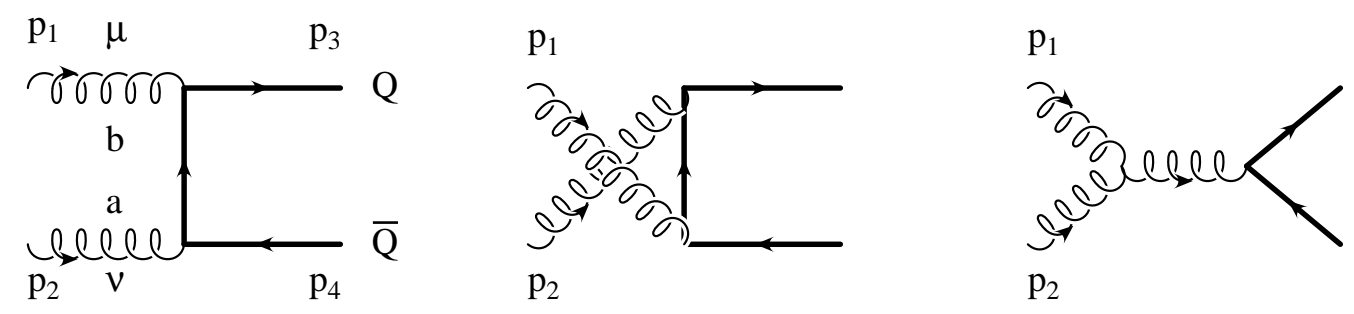

Fig. 1 

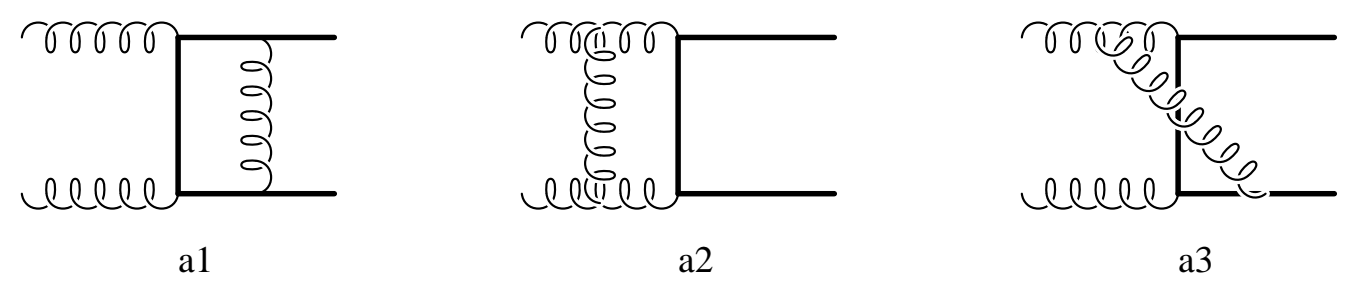

a3

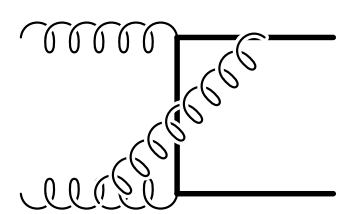

a4
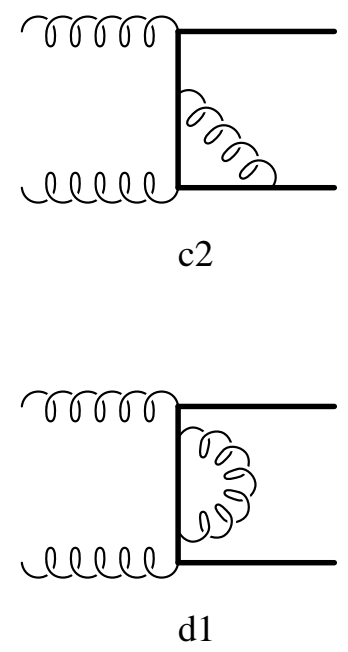

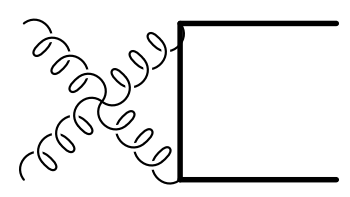

b

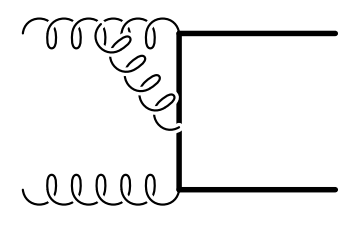

c3

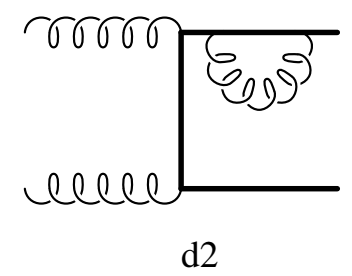

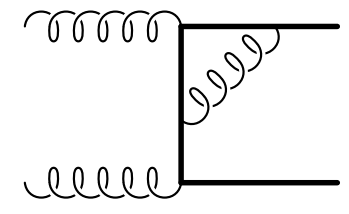

c1
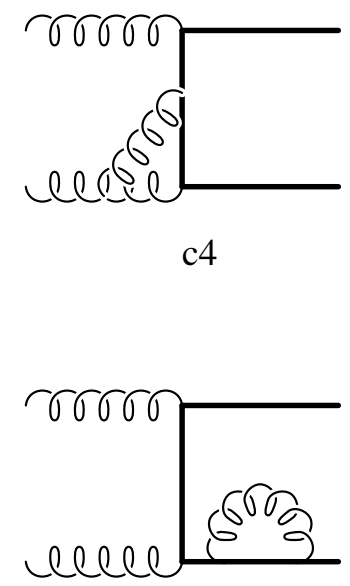

d3
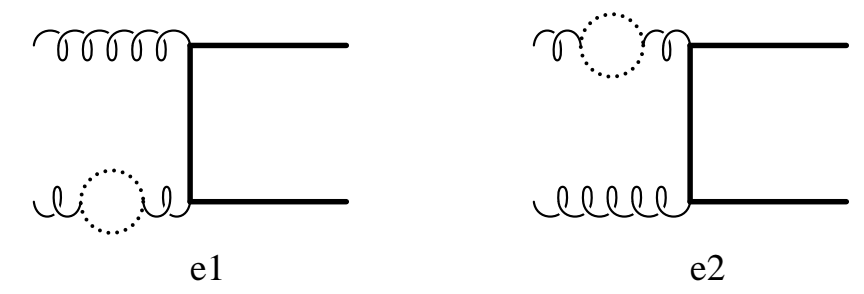

Fig. 2 


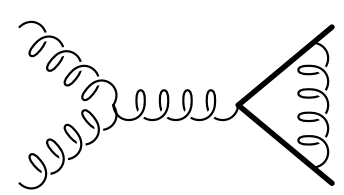

f1

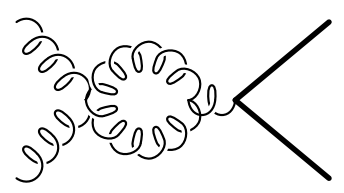

g2

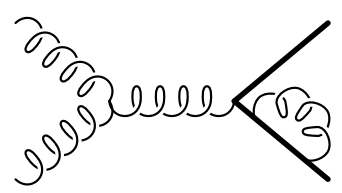

i2

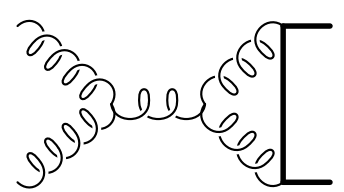

f2

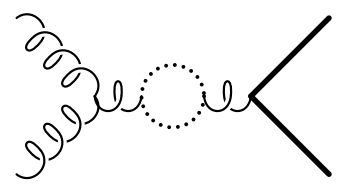

h

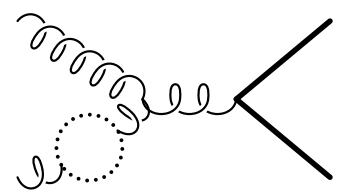

j1

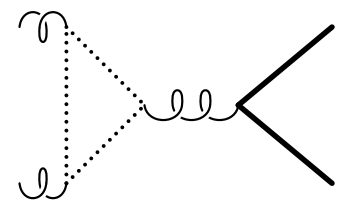

g1

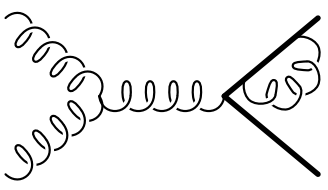

i1

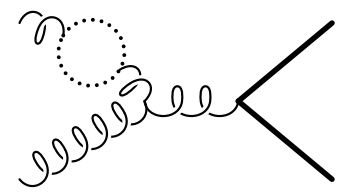

j2

Fig. 3 


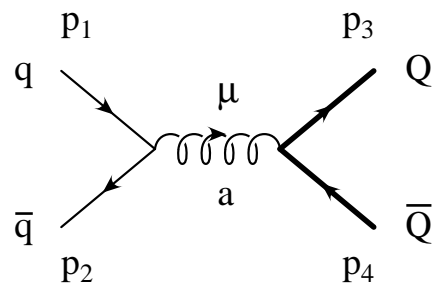

Fig. 4 


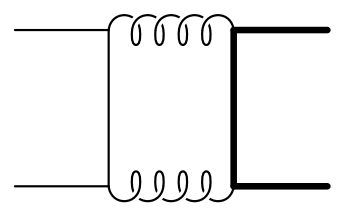

a

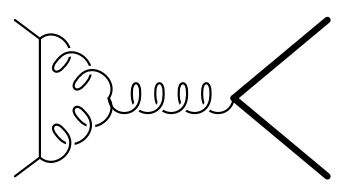

d

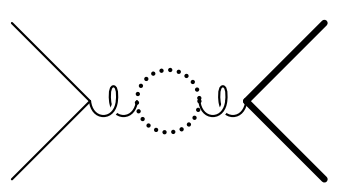

g

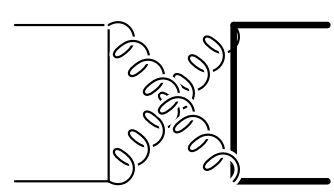

b

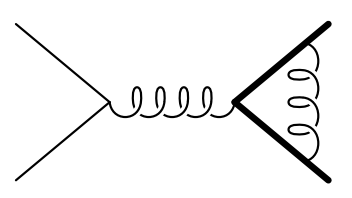

e

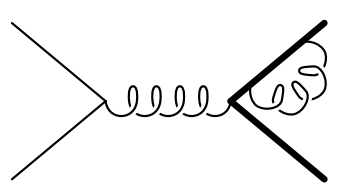

h

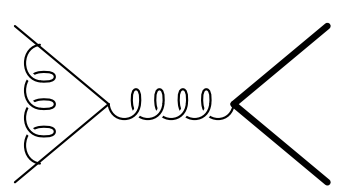

c

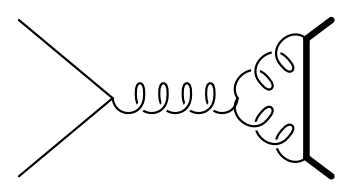

f

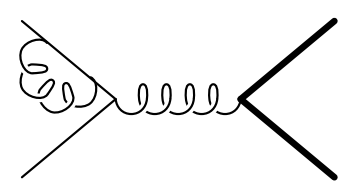

j

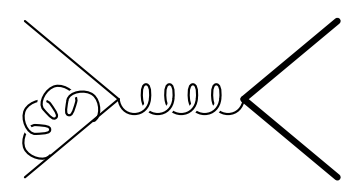

k

Fig. 5 